\title{
The lattice Boltzmann method for isothermal micro-gaseous flow and its application in shale gas flow: a review
}

\author{
Junjian Wang, ${ }^{\mathrm{a}, \mathrm{b}}$, Li Chen ${ }^{\mathrm{c}, \mathrm{b}}$, Qinjun Kang ${ }^{\mathrm{b}}$, Sheik S Rahman ${ }^{\mathrm{a}, *}$ \\ ${ }^{a}$ School of Petroleum Engineering, University of New South Wales, Sydney, \\ NSW,Australia,2033 \\ ${ }^{b}$ Earth and Environmental Sciences Division, Los Alamos National Laboratory, Los \\ Alamos, NM, USA,87545 \\ ${ }^{c}$ Key laboratory of Themo-Fluid Science and Engineering of MOE, School of Energy and \\ Power Engineering, Xi'an Jiaotong University, Xi'an, Shanxi, China, 710049
}

\begin{abstract}
The lattice Boltzmann method (LBM) has experienced tremendous advances and been well accepted as a popular method for simulating various fluid flow problems in porous media. With the introduction of an effective relaxation time and slip boundary conditions, the LBM has been successfully extended to solve micro-gaseous transport phenomena. As a result, the LBM has the potential to become an effective numerical method for gas flow in shale matrix in slip flow and transition flow regimes. Additionally, it is very difficult to experimentally determine the permeability of extremely low permeable media like shale. In this paper an extensive review on a number of slip boundary conditions and Knudsen layer treatments used in LB models for micro-gaseous flow is carried out. Furthermore, potential applications of the LBM in flow simulation in shale gas reservoirs on pore scale and representative elementary volume(REV) scale are evaluated and summarised. Our review indicates that the LBM is capable of capturing gas flow in continuum to slip flow regimes which cover significant proportion of the pores in shale gas reservoirs and identifies opportunities for future research.
\end{abstract}

Keywords: shale, lattice Boltzmann method, micro-gaseous flow, slip flow

\footnotetext{
${ }^{*}$ Corresponding author at: School of Petroleum Engineering, University of New South Wales, Sydney, NSW,Australia,2033

Email address: sheik.rahman@unsw.edu.au (Sheik S Rahman)
} 


\section{Introduction}

Shale gas reservoirs are thought to contain a significant proportion of hydrocarbon, and successful exploitation of such resource plays an increasingly important role in meeting world's demand for natural gas. Shale gas reservoirs are known to be fine grained sedimentary rocks which have complex porous structures with pores and fractures ranging from nano- to mesoscale[1][2], and in each level of pores and fractures different flow mechanisms are involved [3]. An in-depth understanding of flow processes involve in complex porous system in shale is essential for prediction of reservoir permeability and estimating production potential of shale gas reservoirs. This can be achieved by developing detailed descriptive transport simulators which are capable of predicting flow dynamics in shale.

Knudsen number $(K n)$, which is the ratio of the gas mean free path to the characteristic length of the media, is an important dimensionless parameter for gas transport in shale. Current studies conclude that gas transport through shale matrix can best be characterized by $K n$ in slip flow $(0.001<K n<0.1)$ and transition flow $(0.1<K n<10)$ regimes[4][5] (see Fig.1). Under these conditions, continuum hypothesis is broken down and other rarefied gas transport mechanisms such as slip flow and Knudsen diffusion start to dominate the flow. Additionally, as a source rock, the presence of organic matter (kerogen) in shale matrix instigates other processes and adds complexities to gas flow simulation. Gas transport in nano-pores inside the kerogen involves adsorption/desorption as well as surface diffusion due to strong molecular interactions between gas and kerogen.

A variety of experimental and mathematical studies shows that rarefication effects influence the shale permeability measurements by increasing the apparent permeability values[6][7][8][9][10][11][12][13]. The effect of adsorption gas and the following surface diffusion on the permeability of shale, however, is not well understood and less widely explored. On one hand, studies confirmed that the multilayer adsorption can take place in organic pores because of the capillary condensation phenomenon[14][15], which will lead to a lower permeability in shale reservoirs[16]. On the other, it is confirmed that surface diffusion can account for $25 \%$ of total flux at low pressure[17]. Wu et al.[18]stated that when the pore size is less than $2 \mathrm{~nm}$, the contribution of surface diffusion to total mass transfer can be as much as $92.95 \%$. 


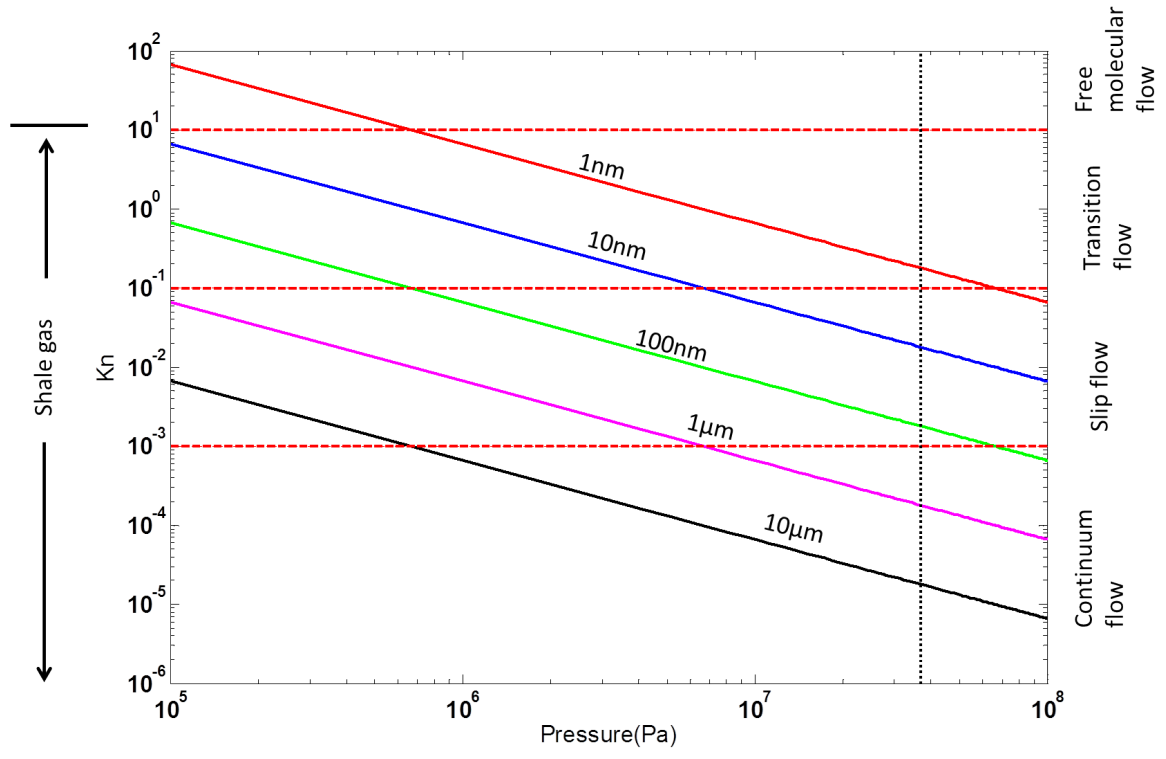

Figure 1: Knudsen number relationship to pore diameter and mean reservoir pressure at $400 \mathrm{~K}$. Vertical dash line represent a typical reservoir pressure condition of $37 \mathrm{MPa}$.(Figure adapted from Javadapour et al.[3] and Sondergeld et al.[2] )

Generally, on the basis of pore size distribution, two possible mathematical approaches are proposed to describe the gas transport mechanism and to calculate gas apparent permeability of organic shales. The first approach is to modify the non-slip boundaries in continuum model by accounting for slip boundary conditions. Beskok-Karniadaki[19] derived a unified HagenPoiseuille-type formula to take into account all flow regimes. Later, Civan and coworkers[20][21] and Florence et al.[22] proposed different forms of rarefaction coefficient for Beskok-Karniadaki model. By simply adding the mass transfer of adsorbed gas into Beskok-Karniadaki model, the impact of the adsorption and surface diffusion on gas apparent permeability is studied by Xiong et al.[23]. The second approach is the superposition of various transport mechanisms. Javadpour[3] combined slip flow and Knudsen diffusion into gas flux equation and derived an equation for apparent permeability. Freeman et al.[5] used dusty gas model to account for Knudsen diffusion in shale gas reservoir. Singh et al.[24] combined viscous flow with Knudsen diffusion in their non-empirical apparent permeability model(NAP), and then validated with previous experimental data. Results have shown that the NAP can be used for $K n$ less than unity. Wu et al.[25] further proposed two 
weighted factors for viscous flow and Knudsen diffusion, respectively. The surface diffusion was also coupled in their apparent permeability model.

Most of the above mentioned analytical/semi-analytical studies are originally proposed based on simple geometries such as channels and tubes, and are not suitable for more complex porous media, such as shales. Therefore, the numerical methods of solving transport equations to obtain an estimate for the permeability are attracting more attention. Especially after the current well-established characterization techniques such as BIB-SEM, FESEM, FIB-SEM and micro-CT enable us to identify a variety of pore structures in shale matrix[1][26][27][28][29][30], the detailed rock images further promote the use of image-based numerical simulation tools. Among them, the lattice Boltzmann method (LBM), which is vastly different from traditional computational fluid dynamics(CFD) methods, has proven to be an effective flow simulation choice in porous media, as the geometry definition in LB model is reduced to defining nodes as being either fluid or solid, which allows the complex pore structure to be modelled with ease and allows for flexibility of the parallelization.

Historically, the LBM was derived from lattice gas automata[31][32]. Later, it was shown that LB equation can also be directly derived by discretising the Boltzmann equation[33][34]. Shortly after its introduction, the LBM became an attractive technique to study single/multi-phase flow [35] [36] [37][38][39] and reactive transport[40][41] in porous media, covering groundwater flow[42], fabric materials[43] and fuel cells[44][45] etc. Detailed pore structure obtained by FIB-SEM and micro-CT have made the LBM a popular alternative to direct numerical solution of the Navier-Stokes equation for flows in tight rocks[46][47][48][49]. Because of its kinetic nature, the LBM is, however, far more than just a N-S solver on pore scale[50]. Advances in micro electrical mechanical system (MEMS) and nanotechnology have spurred interest in the use of the LBM for simulation of microfluidics and tremendous efforts have been made to advance the LBM since 2002. One noteworthy advance is its extension to simulation of gaseous flows in slip flow regime [51][52][53][54][55][56][57][58]. Advances of the LBM have also allowed us to simulate fluid flow in single capillary in transition flow regime[59][60][61][62][63]. In previous studies, most of the micro-gaseous flow was based on single relaxation time (SRT) model[52][55][56][57][59][64][65][66]. Luo[67] argued that slip velocity predicted by SRT is merely an artefact at the solid boundaries. For this reason, other LB models, such as multiple relaxation times (MRT)[60][62], two relaxation times (TRT)[68][69] and 
Filter-matrix lattice Boltzmann (FMLB)[61] were proposed. Results of these studies are in good agreement with that of the benchmark studies including force-driven Poiseuille flow, pressure driven Poiseuille flow, and planar Couette flow. For example, by incorporating the Bosanquet-type effective viscosity and applying slip boundary conditions, Li et al.[62] successfully used MRT models to simulate micro-channel gas flow at $K n$ of up to 3 . This also gives a similar result as that of MRT model with a Stops expression of effective viscosity proposed by Guo et al[60]. Most Recently, Zhuo and Zhong [61] developed a Filter-matrix Boltzmann model with Bosanquet-type effective viscosity and produced reasonable results for micro-channel flow at $K n$ of up to 10.

The rapid growth in unconventional gas, especially shale gas demands a deeper understanding of the physics of fluid flow at the nanoscale to microscale. As a mesoscale method, the LB approach is an effective means of dealing with flow problems whose scales are too small for the continuum mechanics and too large for molecular methods. Although there have been several excellent reports and reviews discussing the LBM for micro-gaseous flow[70][71][72], the particular applications and strengths of LBM in simulating gas flow in shale gas reservoir have not been well addressed. In this review, we briefly introduce some ideas and equations fundamental to the LBM. We also introduce the typical slip boundary conditions and Knudsen layer treatments used in the LB algorithms for micro-gaseous flow, which may be useful in shale gas flow simulation. Finally, we focus on recent development in LB theory and applications for gas flow in shale, the feasibility of these applications are also demonstrated.

\section{Lattice Boltzmann method for isothermal micro-gaseous flow}

In general, the lattice Boltzmann equation with a body force term can be written as[73]:

$$
f_{i}\left(\mathbf{x}+\mathbf{c}_{i} \delta t, t+\delta t\right)-f_{i}(\mathbf{x}, t)=\Omega_{i}\left(f_{i}\right)+\delta t \mathbf{F}_{i},
$$

where $\mathbf{c}_{i}$ indicates the finite number of discrete velocities of particles, $f_{i}$ is the distribution function of particles with speed $\mathbf{c}_{i}, \Omega_{i}$ is the collision term, $\mathbf{F}_{i}$ is the force term which is defined according to the collision operator, $\delta x$ is the uniform lattice spacing and $\delta t$ is the time between two simulation iterations. 
The microscopic variables (density and velocity) are defined as:

$$
\begin{aligned}
\rho(\mathbf{x}, t) & =\sum_{i} f_{i}(\mathbf{x}, t), \\
\rho(\mathbf{x}, t) \mathbf{u}^{*}(\mathbf{x}, t) & =\sum_{i} f_{i}(\mathbf{x}, t) \mathbf{c}_{i}+\frac{\delta t}{2} \mathbf{F} .
\end{aligned}
$$

The most popular velocity sets for two dimensions and three dimensions are given in Fig.2(a) and Fig.2(b), respectively. When one considers applications to micro-gaseous flow, some multi-speed or higher-order LB models such as D2Q21(Fig.2(c))[74] and D3Q39(Fig.2(d))[71][75] have also been developed to increase the order of accuracy in the discretization of velocity phase space.

(a)

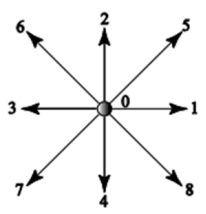

(c)

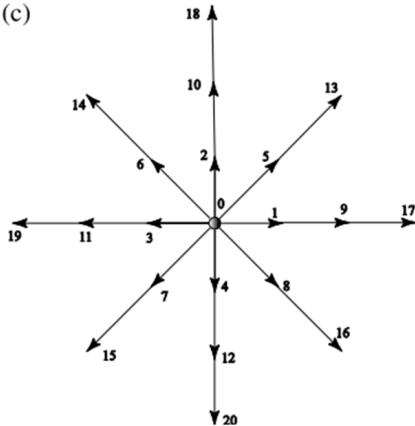

(b)

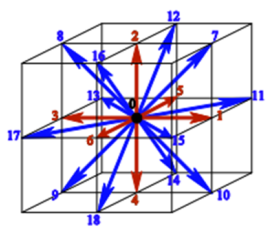

(d)

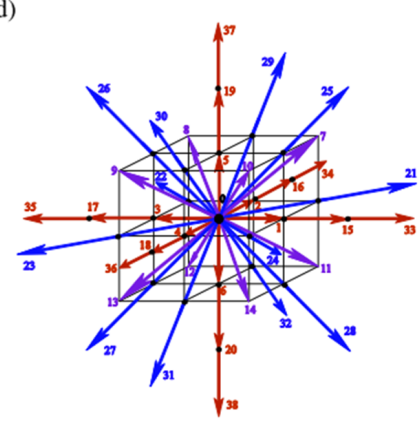

Figure 2: Discrete velocity models; (a)D2Q9 model, (b) D3Q19model, (c) D2Q21 model, (d) D3Q39 model

\subsection{Collision operators}

The collision operators describe the collision behaviour of particles at every lattice location, which represents the variation of distribution functions caused by collision between particles. The requirements are that they should be physically correct and efficiently computable. In the following, collision operators commonly used in micro-gaseous flow are presented. 


\subsubsection{Bhatnagar-Gross-Krook collision operator (BGK)}

Bhatnagar, Gross and Krook [76] proposed an efficient simplification operator to approximate the collision term as:

$$
\Omega_{i}\left(f_{i}\right)=-\tau^{-1}\left(f_{i}-f_{i}^{e q}\right),
$$

where, $\tau$ is the relaxation time and $f_{i}^{e q}$ is the Maxwell Boltzmann distribution of $\mathbf{c}_{i}$ for a given macroscopic fluid velocity $\mathbf{u}$ and density $\rho$. The MaxwellBoltzmann distribution describes the density distribution of a fluid in its equilibrium state to which every fluid strives. The equilibrium distribution $f_{i}^{e q}$ in its discrete form can be expressed as:

$$
f_{i}^{e q}(\mathbf{x}, t)=\rho(\mathbf{x}, t) \omega_{i}\left[1+\frac{3\left(\mathbf{c}_{i} \cdot \mathbf{u}\right)}{c^{2}}+\frac{9\left(\mathbf{c}_{i} \cdot \mathbf{u}\right)^{2}}{2 c^{4}}-\frac{3(\mathbf{u} \cdot \mathbf{u})}{2 c^{2}}\right]
$$

Suga [71] summarized $\mathbf{c}_{i}$, the sound speed $c_{s}$ and the weight parameter $\omega_{i}$ in accordance with each subfigure of Fig.2 (see Table 1).

\begin{tabular}{|c|c|c|c|}
\hline Models & $c_{s}^{2} / c^{2}$ & $\mathbf{c}_{i} / c$ & $w_{i}$ \\
\hline \multirow{3}{*}{ D2Q9 } & \multirow{3}{*}{$1 / 3$} & $(0,0)$ & $4 / 9(i=0)$ \\
\hline & & $( \pm 1,0),(0, \pm 1)$ & $1 / 9(i=1-4)$ \\
\hline & & $( \pm 1, \pm 1)$ & $1 / 36(i=5-8)$ \\
\hline \multirow{6}{*}{ D2Q21 } & \multirow{6}{*}{$2 / 3$} & $(0,0)$ & $91 / 324(i=0)$ \\
\hline & & $( \pm 1,0),(0, \pm 1)$ & $1 / 12(i=1-4)$ \\
\hline & & $( \pm 1, \pm 1)$ & $2 / 27(i=5-8)$ \\
\hline & & $( \pm 2,0),(0, \pm 2)$ & $7 / 360(i=9-12)$ \\
\hline & & $( \pm 2, \pm 2)$ & $1 / 432(i=13-16)$ \\
\hline & & $( \pm 3,0),(0, \pm 3)$ & $1 / 1620(i=17-20)$ \\
\hline \multirow{3}{*}{ D3Q19 } & \multirow{3}{*}{$1 / 3$} & $(0,0,0)$ & $12 / 36(i=0)$ \\
\hline & & $( \pm 1,0,0),(0, \pm 1,0),(0,0, \pm 1)$ & $2 / 36(i=1-6)$ \\
\hline & & $( \pm 1, \pm 1,0),( \pm 1,0, \pm 1),(0, \pm 1, \pm 1)$ & $1 / 36(i=7-18)$ \\
\hline \multirow{6}{*}{ D3Q39 } & \multirow{6}{*}{$2 / 3$} & $(0,0,0)$ & $1 / 12(i=0)$ \\
\hline & & $( \pm 1,0,0),(0, \pm 1,0),(0,0, \pm 1)$ & $1 / 12(i=1-6)$ \\
\hline & & $( \pm 1, \pm 1, \pm 1)$ & $1 / 27(i=7-14)$ \\
\hline & & $( \pm 2,0,0),(0, \pm 2,0),(0,0, \pm 2)$ & $2 / 135(i=15-20)$ \\
\hline & & $( \pm 2, \pm 2,0),( \pm 2,0, \pm 2),(0, \pm 2, \pm 2)$ & $1 / 432(i=21-32)$ \\
\hline & & $( \pm 3,0,0),(0, \pm 3,0),(0,0, \pm 3)$ & $1 / 1620(i=33-38)$ \\
\hline
\end{tabular}

Table 1: Parameters of the discrete velocity models for $2 \mathrm{D} / 3 \mathrm{D}$ 
The forcing term, $\mathbf{F}_{i}$ can be specified with respect to the relaxation parameter $\tau$ and the body force $\mathbf{F}$ as[73]:

$$
F_{i}=\left(1-\frac{1}{2 \tau}\right) \omega_{i}\left[\frac{\mathbf{c}_{i}-\mathbf{u}}{c_{s}^{2}}+\frac{\mathbf{c}_{i} \cdot \mathbf{u}}{c_{s}^{4}} \mathbf{c}_{i}\right] \cdot \mathbf{F} .
$$

Also in order to be valid, the LB equation requires the parameter to fulfil the following relation:

$$
\mu=\rho c_{s}^{2}\left(\tau-\frac{1}{2}\right) \delta t
$$

where, $\mu$ is the dynamic viscosity of the fluid.

\subsubsection{Multiple Relaxation Times collision operator (MRT)}

The MRT-LB model is the most general form within the theoretical framework of the LB equation and kinetic theory. The MRT collision operator relaxes the kinetic moments separately, which can be retrieved from the distribution functions. The MRT collision operator is defined as follows[77]:

$$
\Omega(f)=-\left(\mathbf{M}^{-1} \mathbf{S M}\right)\left(\mathbf{f}-\mathbf{f}^{e q}\right) .
$$

In D2Q9 model, $\mathbf{f}=\left(f_{0}, f_{1}, \ldots, f_{7}, f_{8}\right)^{T}$ denotes the column vector of the distribution functions, $\mathbf{S}$ is the non-negative relaxation matrix:

$$
\mathbf{S}=\operatorname{diag}\left(\tau_{\rho}, \tau_{e}, \tau_{\epsilon}, \tau_{j}, \tau_{q}, \tau_{j}, \tau_{q}, \tau_{s}, \tau_{s}\right)^{-1},
$$

and $\mathbf{M}$ is an orthogonal transform matrix, which maps the distribution functions onto the moment space, and defined as:

$$
\mathbf{M}=\left[\begin{array}{ccccccccc}
1 & 1 & 1 & 1 & 1 & 1 & 1 & 1 & 1 \\
-4 & -1 & -1 & -1 & -1 & 2 & 2 & 2 & 2 \\
4 & -2 & -2 & -2 & -2 & 1 & 1 & 1 & 1 \\
0 & 1 & 0 & -1 & 0 & 1 & -1 & -1 & 1 \\
0 & -2 & 0 & 2 & 0 & 1 & -1 & -1 & 1 \\
0 & 0 & 1 & 0 & -1 & 1 & 1 & -1 & -1 \\
0 & 0 & -2 & 0 & 2 & 1 & 1 & -1 & -1 \\
0 & 1 & -1 & 1 & -1 & 0 & 0 & 0 & 0 \\
0 & 0 & 0 & 0 & 0 & 1 & -1 & 1 & 1
\end{array}\right]
$$

The distribution function and equilibrium function can be projected onto the moment space by using the transform matrix:

$$
\mathbf{m}=\mathbf{M f}=\left(\rho, e, \epsilon, j_{x}, q_{x}, j_{y}, q_{y}, p_{x x}, p_{x y}\right)^{T},
$$


163

$$
\begin{gathered}
\mathbf{m}^{e q}=\mathbf{M} \mathbf{f}^{e q}=\left(\rho, e^{e q}, \epsilon^{e q}, j_{x}, q-x^{e q}, j_{y}, q_{y}^{e q}, p_{x x}^{e q}, p_{x y}^{e q}\right)^{T} \\
=\rho\left(1,-2+3|u|^{2}, 1-3|u|^{2}, u,-v, u,-v, u^{2}-v^{2}, u v\right)^{T},
\end{gathered}
$$

all of these moments have a physically meaningful interpretation: $\rho$ is the density; $e$ is the energy mode; $\epsilon$ is related to the energy square; $j_{x}$ and $j_{y}$ are the $x$ and $y$ components of the momentum; $q_{x}$ and $q_{y}$ correspond to the $x$ and $y$ components of the energy flux, $p_{x x}$ and $p_{x y}$ and are related to the diagonal and off-diagonal component of the stress tensor. $u$ and $v$ are $x$ and $y$ components of velocity $\mathbf{u}$.

The forcing terms, $\mathbf{F}_{i}$ can also be mapped onto the moment space which gives the vector $\overline{\mathbf{F}}$ as:

$$
\overline{\mathbf{F}}=\left(0,6 \mathbf{u} \cdot \mathbf{F},-6 \mathbf{u} \cdot \mathbf{F}, \mathbf{F}_{x},-\mathbf{F}_{x}, \mathbf{F}_{y},-\mathbf{F}_{y}, 2\left(u \mathbf{F}-v \mathbf{F}_{y}\right),\left(u \mathbf{F}+v \mathbf{F}_{y}\right)\right)^{T} .
$$

MRT is able to overcome the deficiencies of the BGK collision operator because it has more degrees of freedom, which can also be used to increase the numerical stability of the method significantly[78].

\subsubsection{Two relaxation time collision operator (TRT)}

The scheme for two relaxation time was developed by Ginzburg [79], for which the collision operator is split into symmetric and anti-symmetric parts as:

$$
\Omega_{i}\left(f_{i}\right)=-\tau_{s}^{-1}\left(f_{i}^{s}-f_{i}^{s e q}\right)-\tau_{a}^{-1}\left(f_{i}^{a}-f_{i}^{a e q}\right),
$$

where relaxation time, $\tau_{s}$ related to shear viscosity, and relaxation time, $\tau_{a}$ related to energy fluxes. The symmetric and anti-symmetric components of distribution function and equilibrium distribution function can be computed as:

$$
f_{i}^{s}=\frac{f_{i}+f_{\bar{i}}}{2}, f_{i}^{s e q}=\frac{f_{i}^{e q}+f_{\bar{i}}^{e q}}{2},
$$

$$
f_{i}^{a}=\frac{f_{i}-f_{\bar{i}}}{2}, f_{i}^{a e q}=\frac{f_{i}^{e q}-f_{\bar{i}}^{e q}}{2} .
$$

The TRT model is comparable with SRT model in the simplicity of implementation and computational efficiency, but retains the advantages of MRT model in terms of accuracy and stability[68][78]. 


\subsection{Capture gas-solid interfacial slip}

In traditional fluid mechanics, the assumption of non-slip at a solid boundary is used as the boundary condition. The non-slip assumption, however, breaks down at micro- and nanoscales[80][81]. Generally, the interfacial slip is generated by hydrophobicity in liquid flow and by Knudsen effect for gas flow [82]. To capture the liquid-solid interfacial slip, the Shan-Chen interparticle potential model is usually used, where the solid-liquid interaction is modelled via an explicit solid-liquid intermolecular potential to predict behaviours at the interface[82][83][84]. This approach appears to have a sound physical basis. However, it may face with more restrictive constraints in actual practice[53] and no definitive results have been provided to demonstrate its ability to capture gas slip in transition flow regime[85].

To capture the gas slip at the solid boundary, single phase LB model is widely used, and the slip boundary condition is adopted to implicitly consider the solid-fluid interaction. Tremendous efforts have been devoted to develop accurate and efficient boundary schemes for the LBM. In the following section we summarize typical boundary conditions that could be useful for micro-gas simulations.

\subsubsection{Bounce-back boundary condition(BB)}

The half way bounce-back scheme [86] is typically utilized for its simplicity and its second-order accuracy. This boundary condition assumes that a particle which collides with the wall is reflected in opposite direction, which means that its momentum is reversed. In the implementation, particles leaving a boundary fluid node $x$ bounce back from the boundary to the original site in the reversed lattice velocity, this behaviour can be described by Eq. 17 as:

$$
f_{i}^{b b}(\mathbf{x}, t+\delta t)=f_{\bar{i}}^{\star}(\mathbf{x}, t),
$$

where $f_{\bar{i}}^{\star}$ denotes the opposing distribution function to $f_{i}$ leaving $x$ after collision at time $t$ such that $c_{i}=-c_{\bar{i}}$. The bounce-back boundary condition was used by Nie et al.[87] to mimic the microscopic flow, however, lately it has been shown that the boundary slip observed from Nie et al.[87] with a pure bounce-back scheme is just a numerical artefact[33] and cannot reflect the physical slip over the surface.

\subsubsection{Specular reflection boundary condition(SR)}

The specular reflection boundary condition is motivated by the observation of elastic collisions between a relatively light particle and a heavy 
boundary, the physical effect of such a collision is that the velocity component, which is orthogonal to the boundary, is reversed. This is described by the following equation for the D2Q9 model:

$$
f_{i}^{s r}(\mathbf{x})=f_{i^{\prime}}^{\star}(\mathbf{x}),
$$

where, $f_{i^{\prime}}^{\star}$ and $f_{i}^{s r}$ are approaching and specular reflecting distribution functions as shown in Fig.3. This boundary condition is applied by Lim et al.[88] to investigate pressure driven and shear driven micro-channel flows, however, it was found that the mesh size has a significant effect on the numerical results in their work[88][89] and pure specular reflection may overestimates the slip velocity[90].

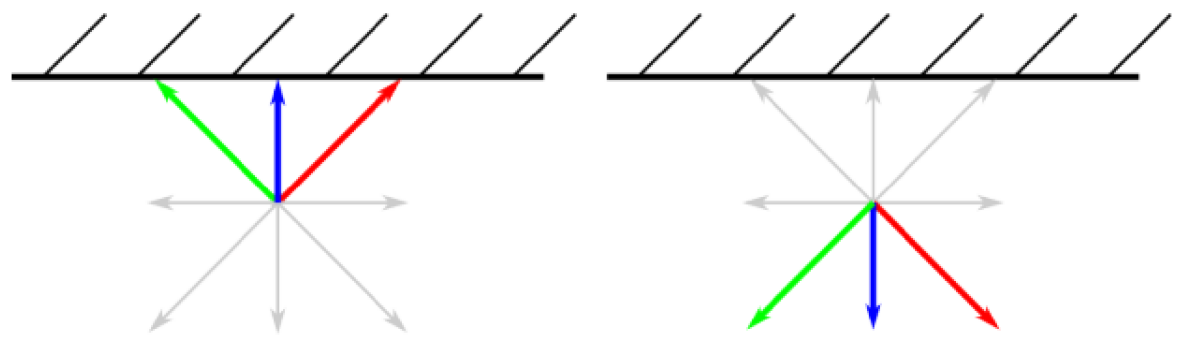

Figure 3: Illustration of the reflection process (in the left image, the distributions before the collision are highlighted. In the right image, the specular reflected distributions after collision with the wall are shown) [91]

\subsubsection{Maxwellian diffuse reflection boundary condition(MD)}

The Maxwellian diffusive reflection boundary condition is derived from the continuum kinetic theory for non-absorbing walls. The underlying idea of this boundary condition is that impinging particles lose the memory of their movement direction and are scattered back following a Maxwellian distribution in which the wall density, $\rho_{w}$ and the wall velocity, $u_{w}$ are known. The fully diffusive boundary condition can be described by the following equation [92]:

$$
f_{i}^{d r}(\mathbf{x})=\frac{\sum_{\left(c_{k}-u_{w}\right) \cdot n<0}\left|\left(c_{k}-u_{w}\right) \cdot n\right| f_{k}^{\star}(\mathbf{x})}{\sum_{\left(c_{k}-u_{w}\right) \cdot n>0}\left|\left(c_{k}-u_{w}\right) \cdot n\right| f_{k}^{e q}\left(\rho_{w}, u_{w}\right)} \cdot f_{i}^{e q},\left(\rho_{w}, u_{w}\right)
$$

with $\left(c_{k}-u_{w}\right) \cdot n>0$. The condition $\left(c_{k}-u_{w}\right) \cdot n<0$ enforces that all incidental distributions are summed up and then redistributed over the 
outgoing distributions such that they obey the equilibrium distribution with mass conservation. The process of the boundary treatment is illustrated in Fig. 4.

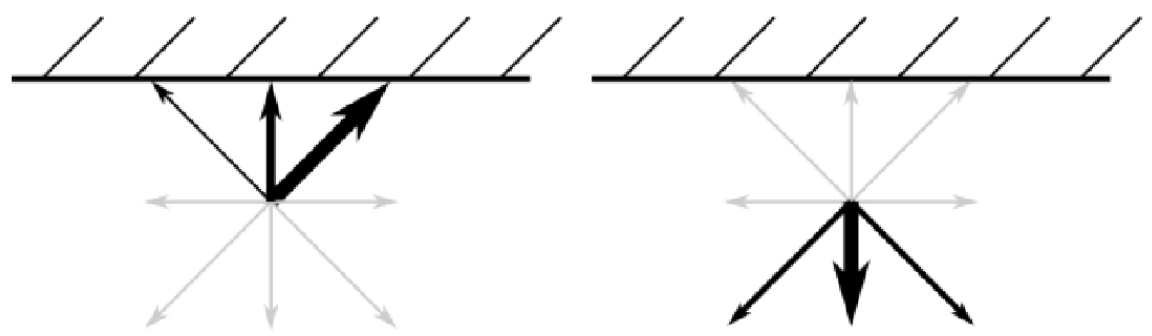

Figure 4: Illustration of the diffuse process(in the left image, the distributions before the collision and in the right image, the diffusive distributions collided with the wall are shown. The thickness of the arrows indicates the value of the distributions)[91]

This boundary condition has been implemented in LB model and the the results show good agreement with the analytical solution of Boltzmann equation for Kramer's problem as the $K n$ tends to be zero[92], the similar concepts are used to simulate micro-gaseous flow at higher $K n$ using D2Q9 LBM [52] and high-order LBM[93].Later Chai et al.[94] argued that the slip velocity is orverpredicted when MD scheme is applied to Poiseuille flow in a micro channel.

\subsubsection{Combined form}

The above mentioned three boundary conditions normally are not directly applied in the LB method, some improved version of the boundary conditions are proposed recently to mimic the macroscopic slip boundary condition by introducing a combination coefficient to control the boundary slip, which includes:

The combined specular with diffusive reflection boundary condition(MR) [66][57][95][96]:

$$
f_{i}(\mathbf{x})=(1-\sigma) f_{i}^{s r}(\mathbf{x})+\sigma f_{i}^{d r}(\mathbf{x}) .
$$

The combined bounce-back with specular reflection boundary condition $(\mathrm{BR})[54]$ :

$$
f_{i}(\mathbf{x})=(1-r) f_{i}^{s r}(\mathbf{x})+r f_{i}^{b b}(\mathbf{x}) .
$$

The combined bounce-back with diffusive reflection boundary condition $(\mathrm{MB})[58]$ :

$$
f_{i}(\mathbf{x})=(1-\chi) f_{i}^{b b}(\mathbf{x})+\chi f_{i}^{d r}(\mathbf{x})
$$


Historically, there is no consensus on how to choose combination coefficients [55][58][61][62][66][97]. Recent studies[58][60][98] indicate that the analytic solution of LBM with bounce-back or combined boundary conditions in Poiseuille flow is just a parabolic profile of N-S equation shifted by a numerical slip, $u_{s}$, and when combined boundary conditions are applied, by setting this numerical slip $u_{s}$ to Maxwellian slip boundary condition, the combination coefficients can be obtained[58][60]. A detailed analysis of three kinds of combined form of boundary conditions are given by Zheng et al.[99] in which they pointed out that if one chooses combination coefficient equal to tangential momentum accommodation coefficient (TMAC)(TMAC $=0.8$ is used in their case studies), the discrete effects of the boundary conditions will induce large numerical errors. Specifically, MR overestimates the slip velocity, while BR and MB underestimate the slip velocity (see Fig.5), and the three boundary conditions cause large errors in the slip velocity ( $>60 \%$ for the BR scheme, $>20 \%$ for the MB scheme, and $>40 \%$ for the MR scheme) within the slip regime (see Fig. 6). For micro-tube flow, the Maxwell-type second order slip boundary can be expressed as:

$$
u_{s}=\left.A_{1} K n \frac{\partial u}{\partial y}\right|_{w a l l}-\left.A_{2} K n^{2} \frac{\partial^{2} u}{\partial y^{2}}\right|_{\text {wall }},
$$

where $u_{s}$ is the slip velocity, $A_{1}$ and $A_{2}$ are the first order and second order slip coefficients, respectively. In order to achieve the second order slip boundary condition, if one chooses the combination coefficients as the following forms (Eq.24 to Eq.26), the three kinds of combined forms are identical in a parametric range, and the discrete effects caused by boundary conditions can be eliminated(see RBR, RMB and RMR in Fig.5 and Fig.6). 

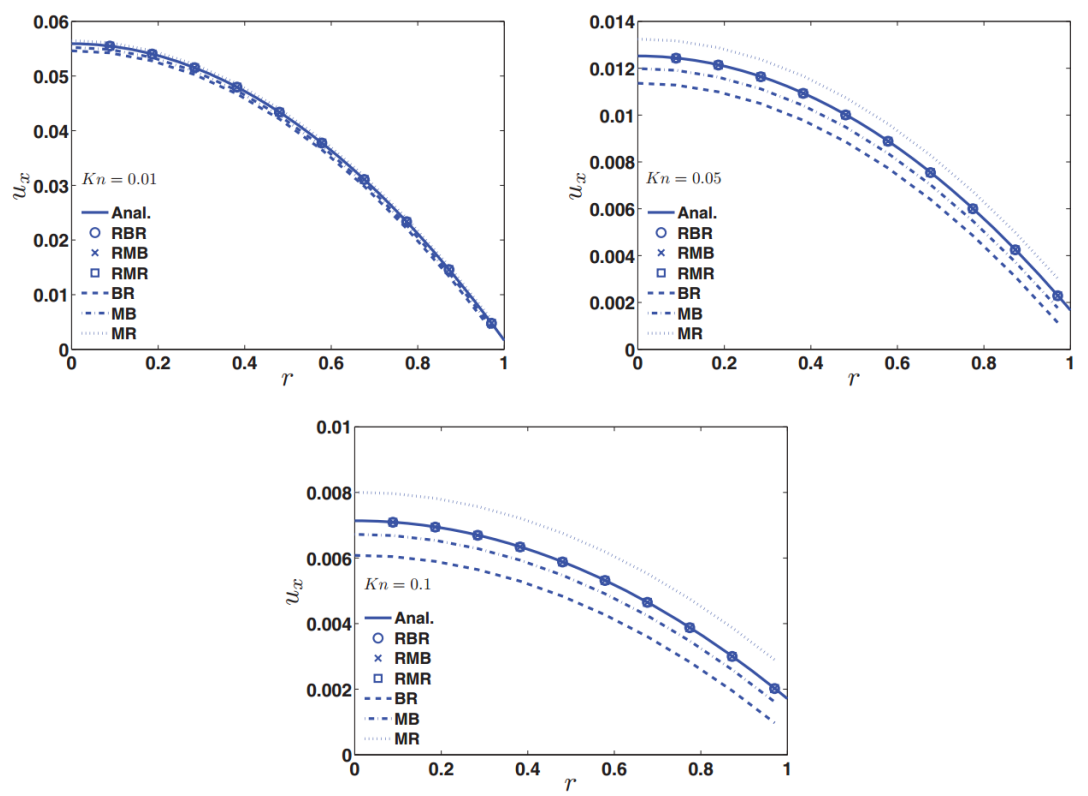

Figure 5: Velocity profiles with TMAC $=0.8[99]$

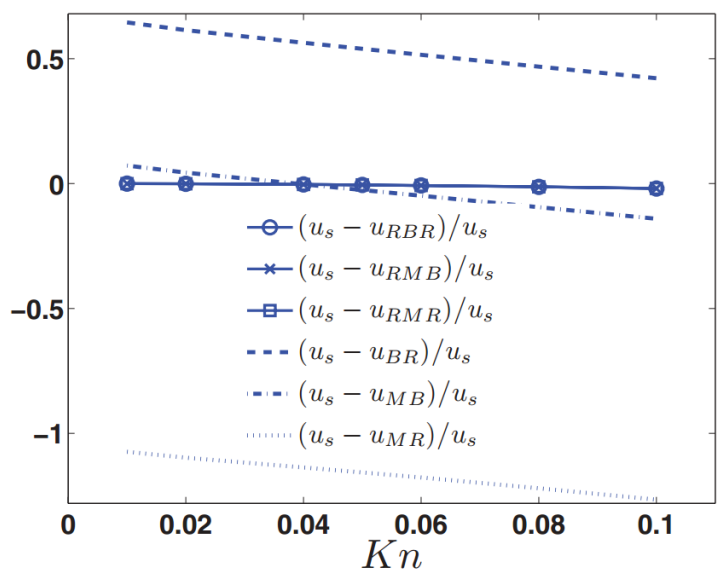

Figure 6: Relative error of velocity slip vs $K n$ with TMAC $=0.8[99]$

$$
\sigma=\left\{1-\sqrt{\frac{\pi}{6}}\left[A_{1}+\left(A_{2}+\frac{8}{\pi}\right) K n\right]+\frac{3 \pi^{1 / 2} \Delta^{2}}{8 \sqrt{6} K n}\right\}^{-1},
$$




$$
\begin{gathered}
r=2-\left\{\frac{1}{2}-\sqrt{\frac{\pi}{6}}\left[\frac{A_{1}}{2}+\left(\frac{A_{2}}{2}+\frac{4}{\pi}\right) K n\right]+\frac{3 \pi^{1 / 2} \Delta^{2}}{8 \sqrt{6} K n}\right\}^{-1}, \\
\chi=\left\{\frac{1}{2}-\sqrt{\frac{\pi}{6}}\left[\frac{A_{1}}{2}+\left(\frac{A_{2}}{2}+\frac{4}{\pi}\right) K n\right]+\frac{3 \pi^{1 / 2} \Delta^{2}}{8 \sqrt{6} K n}\right\}^{-1} .
\end{gathered}
$$

From the above description, LBM can be viewed as an alternative form of slip N-S equation. However, it should be noted that, in Maxwell's original paper, the author used a phenomenological argument to derive slip boundary conditions[100]. This is evident in the fact that the "momentum accommodation coefficients" for each particular gas/surface combination are required in Maxwell-type slip boundary conditions. Typically, the accommodation coefficients can only be inferred from experimental results, rather than directly measured. As a result, the boundary slip observed from combined forms are more phenomenological than physical compared to those from other methods such as DSMC and Boltzmann equation [95] [101], and therefore this fails to demonstrate the physical kinetic nature of the LBM, which has been claimed a distinguishing feature of LBM than other CFD methods. To enable the LBM with combined from boundary conditions to be a predictive tool, Sbragaglia and Succi[53] suggested that a micro-scale simulation, such as MD needs to be used to obtain the values of coefficients corresponding to a given intermolecular potential, prior to the LB simulations at a larger scale which is not accessible by micro-scale simulations.

\subsubsection{Langmuir slip boundary (LSB)}

Another slip boundary used in the LBM is based on Langmuir slip model. The Langmuir slip model has been developed by Eu et al.[102] and Myong [103]. The slip velocity can be expressed as:

$$
u_{s}=\alpha u_{w}+(1-\alpha) u_{g}
$$

where, $u_{g}$ denotes the velocity adjacent to the wall, $\alpha$ is the fraction of surface coved by adsorbed atoms at thermal equilibrium which varies with the type of gas and the nature of wall material. For monatomic gases and diatomic gases $\alpha$ can be expressed by:

$$
\left.\alpha\right|_{\text {mon }}=\frac{\beta p}{1+\beta p} ;\left.\alpha\right|_{d i}=\frac{\sqrt{\beta p}}{1+\sqrt{\beta p}},
$$


with:

$$
\beta=\frac{A \lambda / K n}{k_{b} T} \exp \left(\frac{D_{e}}{K_{b} T}\right)=\frac{1}{4 \omega K n},
$$

where, $k_{b}$ represents the Boltzmann constant, $A$ is the mean area of a site, $D_{e}$ is the potential adsorption parameter and $T$ is the temperature. Compared to Maxwell slip model, in which the slip coefficient is a free parameter from the concept of diffusive reflection, the major advantage of Langmuir slip model is to utilize a physical coefficient of heat and gas particle interaction potential, $\omega[103]$, Nevertheless, Langmuir slip model still has the same difficulty in determining the value of the coefficient as the Maxwell slip model[104].

The LB model with Langmuir slip boundary condition was firstly used to study gas bearing problems[105]. Later, Chen and Tian [106] implement Langmuir slip boundary by non-equilibrium extrapolation scheme to study gas flow in the micro-channel, with an approximation that the local gas density at the wall equals to gas density at the nearby cell, the distribution functions for wall boundary nodes can be expressed as:

$$
f_{i}\left(\mathbf{x}_{b}\right) \approx \alpha\left[f_{i}^{e q}\left(\rho\left(\mathbf{x}_{f}\right), u\left(\mathbf{x}_{b}\right)\right)-f_{i}^{e q}\left(\rho\left(\mathbf{x}_{f}\right), u\left(\mathbf{x}_{f}\right)\right)\right]+f\left(\rho\left(\mathbf{x}_{f}\right), u\left(\mathbf{x}_{f}\right)\right),
$$

where, $\mathbf{x}_{b}$ is the boundary node and $\mathbf{x}_{f}$ is the nearest node to the boundary node.

\subsection{Capture the Knudsen layer effect}

For micro-gaseous flow simulation, Once $K n>0.1$, presence of Knudsen layer near the solid boundary cannot be ignored. Inside of the Knudsen layer, the intermolecular collisions become insufficient and the quasi thermodynamic-equilibrium assumption, upon which the N-S equation depends, does not hold. The standard LBM with a slip boundary condition captures a few low-order moments of the solutions of the Boltzmann equation, and is only accurate at the N-S level[107]. Thus, just like N-S equation, the standard LBM fails to describe the gas motion within the Knudsen layer(See Fig.7). To improve the capability of the LBM for high- $K n$ flows in the transition flow regime, some lattice Boltzmann schemes have been proposed recently. 


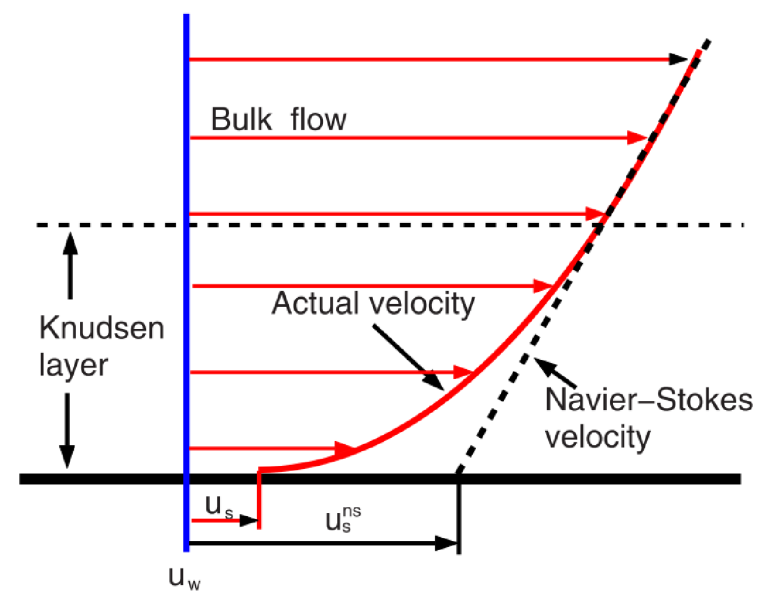

Figure 7: Schematic of the Knudsen layer

\subsubsection{Effective viscosity/mean free path model}

The dynamic viscosity $\mu$ of a fluid plays a critical role in its dynamic behaviour, and thus is an important consideration for fluid flow simulation. The viscosity describes the internal friction between moving fluid layers and the spanwise momentum conductivity. Furthermore, it determines the relaxation time $\tau$ and $\tau_{s}$ used in the BGK-LB model and MRT-LB model, respectively. With an increase in Knudsen number, especially when the mean free path becomes comparable with the characteristic length, the local mean free path is significantly affected by the wall boundaries, which are much smaller than that defined in unbounded systems[95][108]. Since the viscosity and the mean free path are interrelated[109], by introducing an effective relaxation time that corresponds to the effective mean free path or effective viscosity to reflect the gas molecular/wall interactions, the LB models are able to capture the effects of the Knudsen layer for high Knudsen numbers.

\subsubsection{Relations of relaxation time with Kn. By introducing an empirical} parameter $a$, Nie et al.[87] built a linear relationship of $K n$ with relaxation time $\tau$. The problem of this model is that the artificial parameter, $a$ needs to be determined by comparing simulation results with that obtained from experiments, and therefore it cannot be directly used in other situations[52]. To improve this, other attempts have been proposed in the literature, and it has been confirmed that the relaxation time can be related to Kn by multiplying a certain microscopic velocity. The choice of this velocity is rather diverse in the literature(see Table 2), and most recently, Zhang et 
al.[110] and Guo et al.[57] argued that to satisfy a "consistent requirement", this certain microscopic velocity must be chosen as $\sqrt{\pi R T / 2}$, and $\tau$ can be given as:

$$
\tau=H K n /(\delta x \sqrt{\pi / 6})+0.5 .
$$

Table 2: Relationships of relaxation time with $\mathrm{Kn}$

\begin{tabular}{|c|c|}
\hline Nie et al. [87] & $\tau=H K n / a+0.5$ \\
\hline Lim et al. [88] & $\tau=H K n / \delta x$ \\
\hline Lee and Lin [51] & $\tau=H K n / \delta x+0.5$ \\
\hline Niu et al. [52] & $\tau=H K n /(c \sqrt{6 / \gamma \pi}) \approx H K n$ \\
\hline Tang et al. [66] & $\tau=(H K n) /(\delta x \sqrt{8 / 3 \pi})+0.5$ \\
\hline Zhang et al.[56] Guo et al.[57] & $\tau=H K n /(\delta x \sqrt{\pi / 6})+0.5$ \\
\hline
\end{tabular}

2.3.1.2. Wall function approach. One way to capture the Knudsen layer is to modify the mean free path by implementing a "geometry dependent" correction function to reflect the wall confinement as:

$$
\lambda_{e}=\lambda J(r, \lambda),
$$

where, $J(r, \lambda)$ is the correction function which considers the effect of wall surface on the mean free path $\lambda$, and $r$ is distance from the wall. Wall function approach has been widely used to study micro-channel and microtube flows[68][60][63], whist it is a phenomenological approach like that of Maxwell's slip model[111]. Despite the fact that by introducing an effective wall function approach with a more complex collision operator, the LBM can predict the flow behaviour up to the upper end of the transition flow regime[68], the existing wall functions themselves are only accurate in a finite range of $K n$. The different correction functions for parallel walls situations and their valid $K n$ are listed in Table 3. 
Table 3: Summarize of different correction functions

\begin{tabular}{|c|c|c|c|}
\hline & \multicolumn{2}{|c|}{$J$} & $K n$ \\
\hline Stops [112] & $1+\frac{1}{2}$ & $\begin{array}{c}(r / \lambda-1) \exp (-r / \lambda) \\
+((H-r) / \lambda-1) \exp (-(H-r) / \lambda) \\
-(r / \lambda)^{2} E_{i}(r / \lambda) \\
\left.-((H-r) / \lambda)^{2} E_{i}((H-r) / \lambda)\right]\end{array}$ & $\approx 0.2$ \\
\hline Zhang et al. [85] & & $\frac{1}{1+0.7[\exp (-C r / \lambda)+\exp (-C(H-r) / \lambda)]}$ & $\approx 1$ \\
\hline Arlemark et al. [113] & $1-\frac{1}{82}$ & {$\left[\begin{array}{c}\exp \left(-\frac{H / 2+y}{\lambda}\right)+\exp \left(-\frac{H / 2-r}{\lambda}\right) \\
+4 \sum_{i=1}^{7} \exp \left(-\frac{H / 2+r}{\lambda \cos \left[\frac{(2 i-1) \pi}{28}\right]}\right) \\
+4 \sum_{i=1}^{7} \exp \left(-\frac{H / 2-r}{\lambda \cos \left[\frac{(2 i-1) \pi}{28}\right]}\right) \\
\quad+2 \sum_{i=1}^{6} \exp \left(-\frac{H / 2+r}{\lambda \cos \left[\frac{i \pi}{14}\right]}\right) \\
\quad+2 \sum_{i=1}^{6} \exp \left(-\frac{H / 2-r}{\lambda \cos \left[\frac{i \pi}{14}\right]}\right)\end{array}\right.$} & $\approx 0.2$ \\
\hline Dongari et al. [108] & $1-\frac{1}{96}$ & $\begin{aligned} & \left(1+\frac{r}{\lambda}\right)^{-2}+\left(1+\frac{H-r}{\lambda}\right)^{-2} \\
+ & 4 \sum_{i=1}^{8}\left(1+\frac{r}{\lambda \cos \left[\frac{(2 i-1) \pi}{32}\right]}\right)^{-2} \\
+ & 4 \sum_{i=1}^{8}\left(1+\frac{H-r}{\lambda \cos \left[\frac{(2 i-1) \pi}{32}\right]}\right)^{-2} \\
& +2 \sum_{i=1}^{7}\left(1+\frac{r}{\lambda \cos \left[\frac{i \pi}{16}\right]}\right)^{-2} \\
& +2 \sum_{i=1}^{7}\left(1+\frac{H-r}{\left.\lambda \cos s \frac{i \pi}{16}\right]}\right)\end{aligned}$ & $\approx 2$ \\
\hline
\end{tabular}

A general problem of wall functions is that it has been derived based on the distance to the wall and therefore it is difficult to deal with complex geometries and the Knudsen layer overlap effect[63]. To solve this problem, an approximate Stop's expression is proposed by Guo et al.[57] as:

$$
J(K n)=\frac{2}{\pi} \arctan \left(\sqrt{2} K n^{-3 / 4}\right) .
$$

It can be seen that this correction function is not related to the distance from the wall. In other words, with this formulation it is possible to tackle the problem of Knudsen layer interference in more complex geometry by computing the average of all effective mean free paths. 
2.3.1.3. Bosanquet-type effective viscosity approach. The larger the Knudsen number, the closer the mean free path to the characteristic length $H$. In the case $\lambda>>H$, the effective mean free path equals $H$ and the viscosity, $\mu_{\infty}=$ $a_{\infty} \rho \bar{c} H$ with $a_{\infty}$ being a numerical constant. Michalis et al.[114] observed that the effective viscosity can be approximated by the terms $\mu$ and $\mu_{\infty}$. They conducted micro-flow simulations with the Direct Simulation Monte Carlo(DSMC) method to investigate the rarefaction effects on the viscosity in the transition regime. The calculated densities and velocity profiles were used to compute the actual viscosity of the fluid. With these results Michalis et al.[114] showed that the Bosanquet-type of approximation describes quite satisfactorily the Kn-dependence of the viscosity over the entire transition flow regime and it can be expressed as:

$$
\mu_{e} \approx \frac{\mu}{1+a K n},
$$

where, $a$ is the rarefaction factor and $a=a_{0} / a_{\infty}$. Because the Bosanquettype effective viscosity considers the overall rarefaction effect on gas viscosity such as the approximate wall function approach proposed by Guo et al. [57], it can be utilized in rarefied flow simulations in porous media. However, the choice of $a$ is still an open question. Beskok and Karniadakis[19] used $a=2.2$ together with their general slip boundary condition to simulate fluid flow in channels. Later, Sun and Chan[115] used $a=2$ in estimating effective viscosity at different Knudsen number in channels with aspect ratio between 15 and 20. They found the results of effective viscosity is reasonable when compared with that obtained from the DSMC method. Michalis et al.[114] observed that the value of $a$ depends on the Knudsen number and it varies slightly over the majority of the transition flow regime. Recently, Kalarakis et al.[116] suggested $a=3.4$ based on a study in which authors estimated permeability using LB method and then compared the results with that from DSMC for porous media with porosity equal to 0.7 and 0.8 .

\subsubsection{High-order lattice Boltzmann model}

While the effective viscosity/mean free path model is one way of extending slip LB models into the non-equilibrium gas flow, developing high-order LB models has also attracted significant recent interest. Since the LB equation is a discrete approximation to the continuous Boltzmann equation which is capable of describing gas flow in a wide range of Knudsen number, some efforts have been made to design higher-order LB models by increasing discrete 
velocities to achieve its approximation accuracy to the continuous Boltzmann equation[93][107][117][118][119][120].

It has been shown that the high-order LB models can improve the predictions for non-equilibrium flows and the simulation results are qualitatively agree with previous studies[93][107][118], Ansumali et al.[117] presented analytical solutions of the discrete velocity Boltzmann equation for Couette flow using the so-called D2Q9 and D2Q16 schemes and showed that the increase of order in the GaussHermite quadrature results in a much more accurate treatment of finite Kn flows. However, no systematic study on the quadrature set higher than D2Q16 were discussed by them. Later, Kim et al.[74] compared several high-order models including D2Q12, D2Q16, D2Q21, D2Q25 and D2Q36 models, and they concluded that the accuracy of the higher-order LB models does not increase monotonically with the increase of the order of Gauss-Hermite quadrature and cannot guarantee an improved accuracy for microscale gas flow with the Kn up to $\mathrm{O}(1)$. This was further confirmed by Meng and Zhang[121] with a study of D2Q16, D2Q25 D2Q36 D2Q49 and D2Q64 LB models. Kim et al.[74] also reported that the Knudsen layer can be observed with the minimum of D2Q16 discrete velocities even this particular higher-order LB model fails to reproduce the Knudsen minima correctly. In fact, numerical studies suggest that a very high-order LB model is needed to reproduce the Knudsen paradox phenomena[74][121]. In addition to the inconsistencies at higher Kn, large computational costs hinder the applications of high-order LB models in the simulation of more complex flow. Meng and zhang[122] compared the computational costs associated with D2Q25 and D2Q400 models for a quasi-steady standing-shear-wave problem, and they found D2Q400 is more than 240 times slower than D2Q25 while the difference of two simulation results is less than $3 \%$. Recently, Suga and coworkers[71][123][124] compared D2Q9/D3Q19 with D2Q21/D3q39 in the simulation of fluid flow in micro-porous media with a porosity around 0.9 at Kn ranges from 0.04 to 0.24 . They concluded that the flow field results provided by D2Q9/D3Q19 are less accurate than D2Q21/D3Q39. However, the deficiency is not significant in the complex flow fields, and MRT-LB models almost perfectly overlap with those of the high-order LB models, which also means that a higher-order lattice may not be significantly important for predicting general flow profiles in micro porous media. 


\section{Simulation of fluid/gas flow in shale gas Reservoirs with the LBM}

Shale gas reservoirs are considered unique because of its complex petrophysical properties and its inherent multiscale nature, with details at the microscale affecting the overall operation of macroscopic gas production. Generally, theoretical treatments of flow in porous shale are usually associated with three different length scales: pore-(microscopic), representative elementary volume(REV)- and stimulated reservoir volume (SRV)-scales. The SRVscale is the largest scale, which includes tight matrix and multi-scale fracture networks[125]. One of the critical issues in SRV-scale simulation of shale gas reservoir is how to handle fracture flow and fracture/matrix interactions. Compared to LBM, traditional CFD methods such as FEM[126][127][128] and FDM[125][129] are currently primary simulation tools on SRV- scale simulation in dealing with multiple flow mechanism and rock deformation despite the fact that they are still far behind the industry's demand[125]. Pore scale is the smallest scale where the flow is studied on pore geometries. Pore scale results can provide quantities such as permeability, porosity at various locations. With these results, some fundamental issues such as medium variability can be quantitatively assessed and REV can be quantified. The REV scale is larger than the pore scale but much smaller than the field scale, within the range of an REV, the macroscopic variables (such as permeability and porosity) do not change with the magnitude of the averaging volume. As an image based simulation tool, the LBM has been developed to simulate fluid/gas flow in porous shale on pore scale and REV scale.

\subsection{Pore scale simulation}

At the pore scale, the flow of fluid through the pores of shale reservoir is directly simulated by the LBM. Initially, standard LBM is developed with non-slip boundary conditions to study continuum flow in porous media. As gas flow in shale is involved with different flow regimes, recently, some attempts have been made to extend standard LBM to capture non-continuum phenomenon.

\subsubsection{Estimation of intrinsic permeability}

Under the continuum assumption, standard LBM can be easily applied to complex boundary geometries due to its kinetic nature and a simple bounceback rule for non-slip boundary condition. This particular feature makes 
the LBM superior to classical numerical techniques (e.g., finite differences and finite elements) and other simplified network models for studying flow in realistic porous media. Moreover, as the incompressible N-S equation can be obtained in the nearly incompressible limit of the LB model, LBM is widely used to estimate the intrinsic permeability of shale along with tomography techniques[130][131] [132]. For example, Chen et al.[133] generated 3D FIB-SEM images of shale samples and estimated intrinsic permeability and tortuosity with standard LBM. Later the same techniques were applied but the computation was carried out using a pore-scale GPU-accelerated LB simulator (GALBS) which increases the computing speed (1000 times faster than the serial code and 10 times faster than the paralleled code run on a standalone CPU)[134]. The relative permeability of shale was also studied by Cantisano et al.[135] and Nagarajan et al.[136]. Nagarajan et al.[136] calculated the gas-oil relative permeability of Liquid Rich Shale (LRS) with the LBM and compared their results with laboratory studies. The authors observed that standard LBM can give similar remaining oil saturation to that of the experimental observations, however, significant difference exists in the relative permeability curves.

\subsubsection{Estimation of apparent permeability}

When continuum hypothesis breaks down, the gas molecules tend to "slip" on the solid surface, and the measured gas permeability through a porous media is higher than that of intrinsic permeability due to gas slippage. To estimate the gas apparent permeability $\left(k_{a}\right)$ of shale, several approaches have been proposed from the literature.

3.1.2.1. Klinkenberg model based LBM. In Klinkenberg model, the apparent permeability is calculated based on a linear correlation factor $\left(f_{c}\right)$ for correcting the intrinsic permeability $\left(k_{0}\right)$ :

$$
k_{a}=k_{0} f_{c},
$$

where, $f_{c}$ is a correction factor and is given by [137]:

$$
f_{c}=\left(1+\frac{b_{k}}{p}\right)
$$

with a slip factor $b_{k}$ which depends on $K n$. Later, it is confirmed that Klinkenberg's correlation is only first order accurate. Beskok and Karniadakis [19] proposed a second-order correlation by considering the different 
flow regimes from continuum flow to free molecular flow:

$$
f_{c}=[1+\alpha(K n) K n]\left[1+\frac{4 K n}{1-b K n}\right]
$$

where, slip coefficient $b$ equals to -1 for slip flow, and $\alpha(K n)$ is a rarefaction coefficient which is given by Florence et al.[22] for a purely diffusive ( $\mathrm{TMAC}=1$ ) situation as:

$$
\alpha(K n)=\frac{128}{15 \pi^{2}} \tan ^{-1}\left[4 K n^{0.4}\right] .
$$

Allan and Mavko[138] used the combination of Beskok and KarniadakisFlorence's correlation along with a 3D incompressible LB model to estimate the $k_{a}$ for a shale image with a $167 \mathrm{~nm}$ length in each dimension. In their study, the intrinsic permeability $k_{0}$ was predicted by standard LBM. Further, the adsorption gas was induced in their model as an immobile phase which affects permeability in two manners: decreasing the gas permeability and changing the TMAC. Moreover, they pointed out that a supercritical phase transition may take place during the pressure depression and diffusive flow mechanisms becomes a negligible mass transport mechanism when gas is in a supercritical phase (see Fig. 8).

Allan and Mavko's work appears to be the first to apply the LBM to quantify the effect of slip and adsorption on micro-porous shale rock transport properties. With an average $K n$ which is derived from flux weighted average pore width, however, the realistic gas flow through the pore space cannot be obtained. Also, the surface diffusion of adsorption gas is not considered in their model, and no further discussions are provided on the influence of adsorption gas on TMAC. 


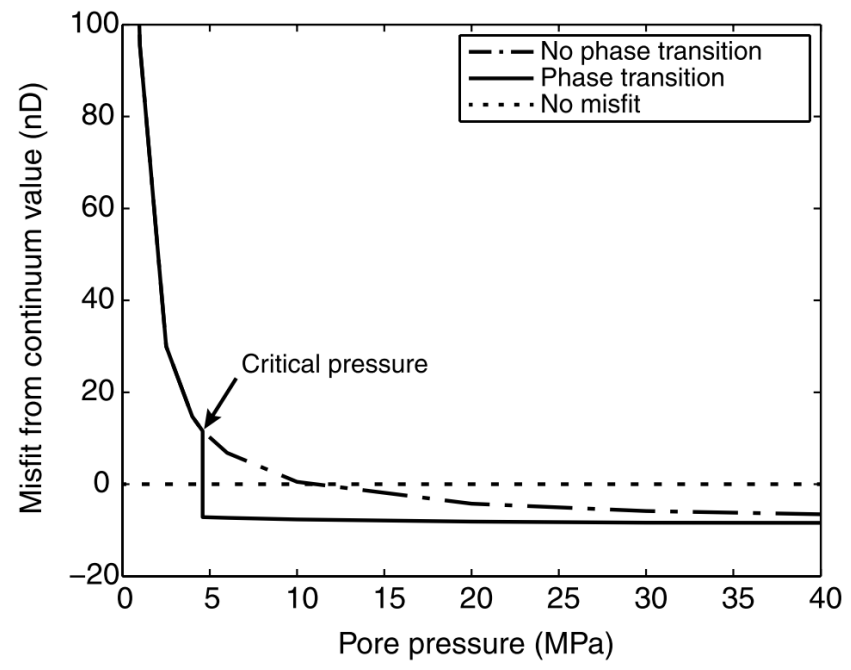

Figure 8: The difference between the total permeability and the continuum permeability as a function of pore pressure. For comparison, two curves are presented corresponding to a gaseous phase (dot-dashed curve), and to a fluid phase that undergoes a supercritical transition (solid curve). The horizontal dotted line marks that the total permeability is equal to the continuum permeability when adsorption layer is not considered.

3.1.2.2. Dusty gas model (DGM) based LBM. The dusty gas model is based on the superposition of convection and molecular spatial diffusion(Knudsen diffusion):

$$
J=-\frac{\rho k_{a}}{\mu}=J_{d}+J_{k}=-\frac{\rho k_{d}}{\mu} \nabla p+-\frac{\rho}{p} D_{e f f} \nabla p
$$

where, $J$ is the mass flux per unit area, $J_{d}$ is the viscous flow flux and $J_{k}$ is the Knudsen diffusion flux. $D_{\text {eff }}$ is the effective Knudsen diffusivity. Based on Eq.39, the apparent permeability can be calculated as:

$$
k_{a}=k_{0}\left(1+\frac{D_{e f f} \mu}{p k_{0}}\right) .
$$

Very recently, a DGM based LB model was proposed by Chen et al.[139] to calculate the apparent permeability of shale. In this model a MRT-LBM for fluid flow and a SRT-LBM for mass transport were used to estimate intrinsic permeability $k_{0}$ and effective Knudsen diffusivity $D_{\text {eff }}$, respectively. In order to account for the variation of local Knudsen diffusivity with local 
pore diameter, the relaxation time used in the LBM transport model was modified based on:

$$
\tau_{g}=\frac{d_{p}}{d_{p, r e f}}\left(\tau_{p, r e f}-0.5\right)+0.5,
$$

where $d_{p}$ is local pore diameter, $d_{p, r e f}$ is a reference pore size which is chosen as $25 \mathrm{~nm}$ in their simulation, and $\tau_{p, r e f}$ is set as 1.0.

The DGM based LB model developed by Chen et al.[139] was adopted to estimate the tortuosity and the gas apparent permeability of four reconstructed shale samples form Sichuan Basin (China). Their simulation results indicate that commonly used Bruggeman equation greatly underestimates tortuosity of shale, and DGM based LB model can give a comparable results of apparent permeability as that given by Beskok and Karniadakis-Civan's correction[140](see Fig.9).

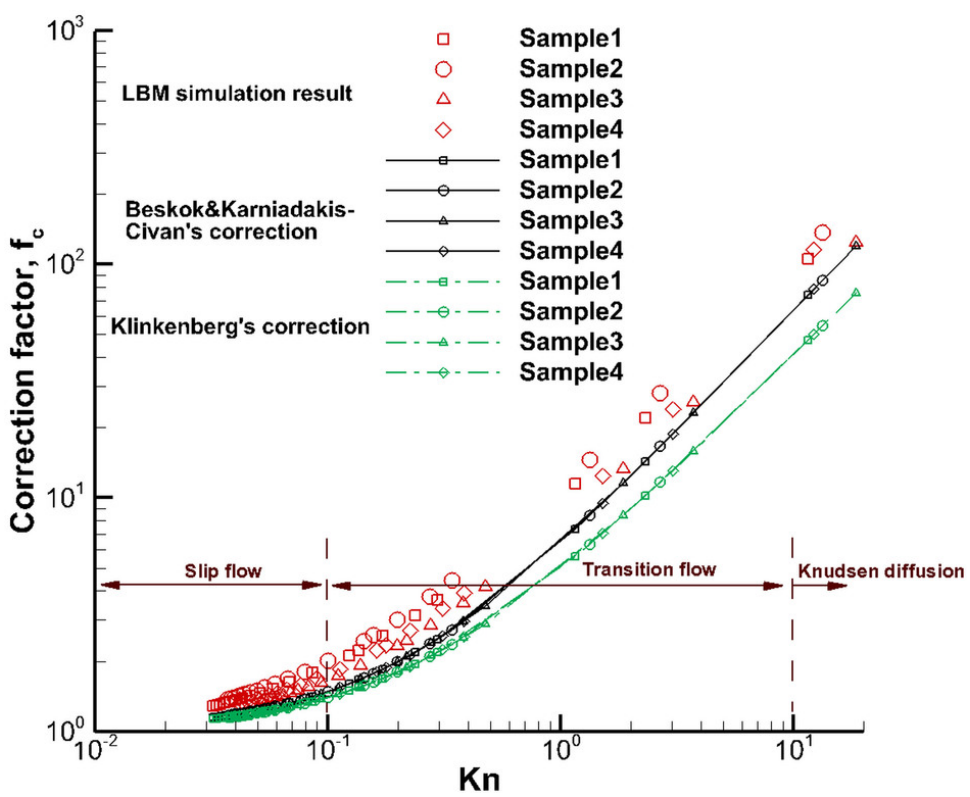

Figure 9: Correction factors predicted by the LBM and empirical correlations under different Knu

Later, the same techniques were applied by them to estimate the apparent permeability of 250 reconstructed kerogen samples using a reconstructed method called overlapping sphere method, with a porosity ranging from 0.1 to 0.55 , and a mean diameter of 30,45 , and $60 \mathrm{~nm}[141]$. The simulation 
results further confirm the high turtuosity of kerogen pores and Beskok and Karniadakis-Civan's correlation for calculating apparent permeability of shale matrix.

To our best knowledge, Chen et al.'s work[139][141] is the first study of numerically investigating the effective Knudsen diffusivity based on real porous structures of shale with the LBM. The advantage of this method is ease of implementation. In their DGM based LBM, gas adsorption and surface diffusion are not considered, and the Knudsen diffusion term used in DGM contains the assumption of fully diffusive boundary condition $(\mathrm{TMAC}=1)$ which underestimates the mass flux in the transition regime[142].

3.1.2.3. Slip $N-S$ model based $L B M$. Slip N-S model based LBM is the direct simulation of gas flow through pore structure with the use of appropriate slip boundary conditions and effective relaxation time(See section 2). A few attempts have been made in the literature by applying slip LBM to shale gas flow through kerogen pores. In these applications, additional physical properties are added into slip boundary conditions and LB model to reflect the adsorption gas and/or surface diffusion effect.

Fathi and Akkutlu [143] developed a LB-Langmuir isotherm model in which LSB was used to capture the velocity slip. To consider the impact of surface diffusion, $u_{w}$ in Eq.27 was calculated based on:

$$
u_{w}=u_{a}=\frac{D_{s}}{D_{k} \rho_{a}}\left[\rho_{g} \mu_{g} \frac{K C_{\mu s}}{(1+K C)^{2}}\right],
$$

where, $C$ is the free gas density, $C_{\mu s}$ is the maximum adsorption capacity, $D_{k}$ is the tortuosity-corrected coefficient of molecular diffusion, $D_{s}$ is surface diffusion coefficient, $\rho_{a}$ is the adsorbed-phase density, and $K$ is the equilibrium partition (or distribution) coefficient. Interestingly, the pseudopotential model proposed by Shan and Chen[144] was also employed in their LB model to consider the non-ideal gas effect and the interactions between solid and gas, however, it is argued that this treatment may lead to a double consideration of the gas-solid interactions[145] as pseudopotential model and slip LB are parallel in capturing gas slippage[82][83][84].

Based on BR and Langmuir isotherm model, Ren et al.[146] proposed a different form of LB slip boundary condition, in which the surface diffusion of adsorption gas is modelled as a moving wall. In their model, the transport rate of adsorbed gas is independent of $D_{k}$ as mentioned in Eq.42 and can be 
601

expressed as:

$$
u_{a}=-D_{s} \frac{\rho_{s} M}{\rho_{a} V_{s t d}} \frac{q_{L} p_{L}}{\left(p_{L}+p_{\text {free }}\right)^{2}} \frac{\partial p_{\text {free }}}{\partial x},
$$

where, $M$ is the molecular weight of the gas, $\rho_{s}$ is the organic solid density, $V_{s t d}$ is the gas volume per mole at standard temperature and pressure, $q_{L}$, $p_{L}$ and $p_{\text {free }}$ are Langmuir volume, Langmuir pressure, and free-gas pressure, respectively. They demonstrated that their model can predict more reasonable physical behaviour compared to that in Fathi and Akkutlu[143], however, no validation is provided when considering the adsorption gas and surface diffusion effect. The non-ideal gas effect was also studied by Ren et al.[146](see Fig.10), and they demonstrated the necessity to use LB under real gas conditions instead of the one under ideal gas conditions as large difference exists between the simulation results for ideal and non-ideal gas.

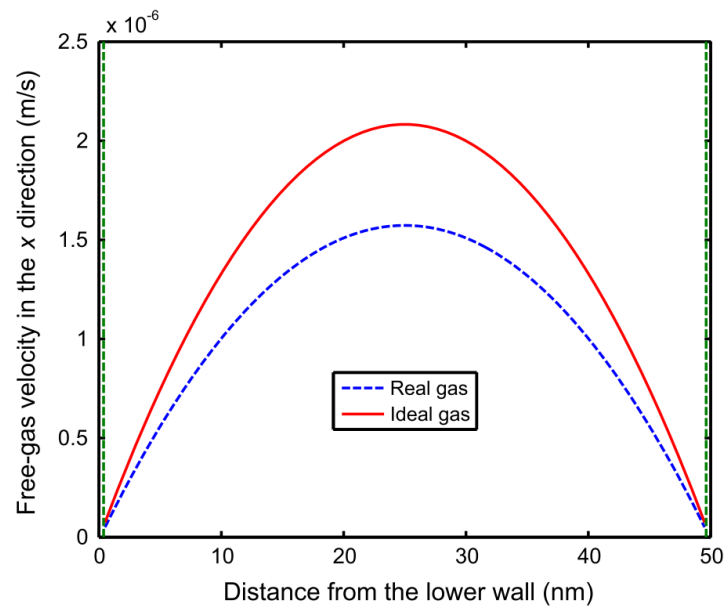

Figure 10: Comparison of free-gas velocity profiles in Kerogen pores using the LB under ideal and non-ideal gas conditions

Slip N-S based LBM gets rid of the usage of empirical or semi-empirical correlations, and it is believed that it can provide accurate simulation results comparable with experimental studies. The main advantage of this method is that detailed local information of the flow (such as velocity field and pressure field) can be obtained and the local information can be used to study macroscopic relations. The slip N-S based LBM is, however, still in its infancy, and its applications in shale are limited to single channel flow. 
Also current complex boundary treatments prevent its further application in porous media[82][94].

\section{2. $R E V$ scale simulation}

Due to the huge computational cost, pore-scale LBM is impractical to perform REV-scale simulations of porous shale. Therefore, several alternative approaches are proposed to apply the LBM on REV scale[36][147][148][149].

To apply the LBM for REV scale flow simulation, Guo et al.[36] proposed a generalized LB model, in which the forcing term(Eq.6) was related to the porosity of porous media:

$$
F_{i}=\left(1-\frac{1}{2 \tau}\right) \omega_{i}\left[\frac{\mathbf{c}_{i}-\mathbf{u}}{c_{s}^{2}}+\frac{\mathbf{c}_{i} \cdot \mathbf{u}}{\varepsilon c_{s}^{4}} \mathbf{c}_{i}\right] \cdot \mathbf{F}
$$

with

$$
\mathbf{F}=-\frac{\varepsilon \mu}{k_{0}} \mathbf{u}-\frac{\varepsilon \rho F_{\varepsilon}}{\sqrt{k_{0}}}|\mathbf{u}| \mathbf{u}+\varepsilon \rho \mathbf{G}
$$

where, $F_{\varepsilon}$ is the geometry function and $k_{0}$ is the intrinsic permeability. Both $F_{\varepsilon}$ and $k_{0}$ need to be estimated based on the empirical relationships with porosity, $\varepsilon$.

Chen et al.[150] further extended this generalized LBM for slip flow by considering Klinkenberg's effect on REV scale, and this was achieved by the usage of apparent permeability, $k_{a}$ instead of $k_{0}$. Beskok and KarniadakisCivan's correlation [20] was adopted to calculate $k_{a}$ :

$$
k_{a}=k_{0}\left[1+\frac{1.358}{1+0.17 K n^{-0.4348}} K n\right]\left[1+\frac{4 K n}{1+K n}\right],
$$

where, $k_{0}$ is calculated based on Kozeny-Carman (KC) equation[151], and the local characteristic pore radius for the calculation of $K n$ was estimated by[152]:

$$
r=0.08886 \sqrt{\frac{k_{0}}{\varepsilon}} .
$$

Chen et al.[150] performed several analysis based on a heterogeneous shale matrix with natural fractures, organic matter and inorganic minerals., Later the same technique were used by them to quantify the permeability of reconstructed shale matrix[153]. Their simulation results qualitatively and quantitatively confirm the increase in permeability induced by Klinkenberg's 
effect. Moreover, Chen et al's study provides a framework of applying the LBM on REV scale for further research, within this framework, other physical effects (such as adsorption and surface diffusion) can be easily integrated by modifying the local apparent permeability.

\section{Discussions and Conclusions}

The LB method has gone through significant improvements over the years and has become a viable and efficient substitute for N-S solver for many flow problems. Because of the underlying kinetic nature, LB equation has attracted a huge interest in its extension to mimic micro-gaseous flows. In this paper we presented a general review of the LBM with an emphasis on boundary conditions, treatments for relaxation time and application of the LBM in isothermal fluid/gas flow simulation in shale gas reservoir.

It has been found that the LBM is an effective method for simulating micro gas flow in continuum to slip flow regime. For example, through the Chapman-Enskog expansion, lattice Boltzmann equation can be proved to recover the macroscopic continuity and momentum (N-S) equations. In slip flow regime, the Knudsen layer takes a small portion of the channel height, which can be neglected by extrapolating the bulk gas flow towards the wall. In this case, with the implementation of proper relaxation time and combination coefficients for various boundaries such as BB, SR or MD, the LB equation can give similar results to that of N-S equation with slip boundary conditions for pressure driven/force driven micro-channel flow and microCouette flow.

In transition flow regime, the Knudsen layer effect is significant, and the N-S equation with first-order slip boundary breaks down. Second-order or high-order slip boundary conditions are needed, and the change of the local mean free path/Knudsen number inside of the Knudsen layer has to be considered. The validation with MD or DSMC simulation results indicates that the LBM can be extended to simulate gas flow in transition flow regimes with high-order velocity sites and/or using wall function approach or approximated viscosity approach. However, because current effective viscosity models are only accurate with a moderate range of Knudsen number and the degree of freedom in the momentum space is very limited, difficulties still exist in studying non-equilibrium gas flow with high Knudsen numbers using LBM. 
Several LB approaches are proposed to study gas transport in shale gas reservoir both on pore scale and REV scale. Pore scale simulation is an effective way to improve the understanding of different flow mechanisms. Application of the LBM to study shale gas transport on pore scale depends very much on its ability to capture micro-gaseous flow and the transport of adsorption gas. Slip N-S based LBM has the potential to provide accurate simulation results comparable with experimental studies with small Knudsen number. Existing slip N-S based LB models are premature to accurately estimate micro-flow properties in porous shale, and Klinkenberg model or DGM based LBM provide an alternative way to simulate gas flow on pore scale. The development of REV scale based LBM enable us to simulate gas flow on a larger scale, however, the simulation study should be complemented by laboratory studies on core samples. Increase in efficiency of these methods for simulation of micro-gaseous flow have the potential for applications in evaluating shale gas reservoirs.

The LBM still has a number of limitations in studying micro-gaseous flow, such as capturing the gas-solid interactions and non-ideal gas effect. Compared to other methods such as MD and DSMC, most of the previous applications of the LBM are more phenomenological than physical. For gas flow in shale gas reservoir, studies based on experiments, MD and DSMC are recommended to be carried out before using the LBM.

\section{Acknowledgement}

The authors would like to acknowledge the support from the LANL's LDRD Program and Institutional Computing Program and SCOPE, UNSW. J.W. would like to acknowledge the support from China Scholarship Council(CSC), L.C. thanks the support from National Nature Science Foundation of China(No. 51406145 and 51136004), and Q.K. would also like to acknowledge the support from a DOE oil \& gas project.

\section{References}

[1] R. G. Loucks, R. M. Reed, S. C. Ruppel, D. M. Jarvie, Morphology, genesis, and distribution of nanometer-scale pores in siliceous mudstones of the mississippian barnett shale, Journal of Sedimentary Research 79 (12) (2009) 848-861. 
[2] C. H. Sondergeld, K. E. Newsham, J. T. Comisky, M. C. Rice, C. S. Rai, Petrophysical considerations in evaluating and producing shale gas resources, in: SPE Unconventional Gas Conference, Society of Petroleum Engineers, 2010.

[3] F. Javadpour, D. Fisher, M. Unsworth, Nanoscale gas flow in shale gas sediments, Journal of Canadian Petroleum Technology 46 (10) (2007) $55-61$.

[4] S. M. Kang, E. Fathi, R. J. Ambrose, I. Y. Akkutlu, R. F. Sigal, Carbon dioxide storage capacity of organic-rich shales, SPE Journal 16 (04) (2011) 842-855.

[5] C. Freeman, G. Moridis, T. Blasingame, A numerical study of microscale flow behavior in tight gas and shale gas reservoir systems, Transport in Porous Media 90 (1) (2011) 253-268.

[6] D. Luffel, C. Hopkins, S. P.D., Matrix permeability measurement of gas productive shales, in: SPE Annual Technical Conference and Exhibition, Society of Petroleum Engineers, 1993.

[7] A. Tinni, E. Fathi, R. Agarwal, C. H. Sondergeld, I. Y. Akkutlu, C. S. Rai, Shale permeability measurements on plugs and crushed samples, in: SPE Canadian Unconventional Resources Conference, Society of Petroleum Engineers, 2012.

[8] J. Zhang, G. W. Scherer, Permeability of shale by the beam-bending method, International Journal of Rock Mechanics and Mining Sciences 53 (2012) 179-191.

[9] Y. M. Metwally, C. H. Sondergeld, Measuring low permeabilities of gassands and shales using a pressure transmission technique, International Journal of Rock Mechanics and Mining Sciences 48 (7) (2011) 11351144 .

[10] A. Ghanizadeh, A. Amann Hildenbrand, M. Gasparik, Y. Gensterblum, B. M. Krooss, R. Littke, Experimental study of fluid transport processes in the matrix system of the european organic-rich shales: Ii. posidonia shale (lower toarcian, northern germany), International Journal of Coal Geology 123 (2014) 20-33. 
[11] R. Heller, J. Vermylen, M. Zoback, Experimental investigation of matrix permeability of gas shales, AAPG Bulletin 98 (5) (2014) 975-995.

[12] A. Mehmani, M. Prodanović, F. Javadpour, Multiscale, multiphysics network modeling of shale matrix gas flows, Transport in Porous Media 99 (2) (2013) 377-390.

[13] Y. Yuan, N. G. Doonechaly, S. Rahman, An analytical model of apparent gas permeability for tight porous media, Transport in Porous Media (2015) 1-22.

[14] B. Li, A. Mehmani, J. Chen, D. T. Georgi, G. Jin, The condition of capillary condensation and its effects on adsorption isotherms of unconventional gas condensate reservoirs, in: SPE Annual Technical Conference and Exhibition, Society of Petroleum Engineers, 2013.

[15] J. Chen, A. Mehmani, B. Li, D. Georgi, G. Jin, Estimation of total hydrocarbon in the presence of capillary condensation for unconventional shale reservoirs, in: SPE Middle East Oil and Gas Show and Conference, Society of Petroleum Engineers, 2013.

[16] G. Jin, H. G. Pérez, G. Agrawal, M. R. Khodja, A. Z. Ali, S. R. Hussaini, Z. Z. Jangda, The impact of gas adsorption and composition on unconventional shale permeability measurement, in: SPE Middle East Oil \& Gas Show and Conference, Society of Petroleum Engineers, 2015 .

[17] L. Zhang, D. Li, D. Lu, T. Zhang, A new formulation of apparent permeability for gas transport in shale, Journal of Natural Gas Science and Engineering 23 (2015) 221-226.

[18] K. Wu, X. Li, C. Wang, W. Yu, Z. Chen, Model for surface diffusion of adsorbed gas in nanopores of shale gas reservoirs, Industrial \& Engineering Chemistry Research 54 (12) (2015) 3225-3236.

[19] A. Beskok, G. E. Karniadakis, Report: a model for flows in channels, pipes, and ducts at micro and nano scales, Microscale Thermophysical Engineering 3 (1) (1999) 43-77.

[20] F. Civan, Effective correlation of apparent gas permeability in tight porous media, Transport in Porous Media 82 (2) (2010) 375-384. 
[21] F. Civan, C. S. Rai, C. H. Sondergeld, Shale gas permeability and diffusivity inferred by improved formulation of relevant retention and transport mechanisms, Transport in Porous Media 86 (3) (2011) 925944.

[22] F. A. Florence, J. Rushing, K. E. Newsham, T. A. Blasingame, Improved permeability prediction relations for low permeability sands, in: Rocky Mountain Oil \& Gas Technology Symposium, Society of Petroleum Engineers, 2007.

[23] X. Xiong, D. Devegowda, M. Villazon, G. German, R. F. Sigal, F. Civan, A fully-coupled free and adsorptive phase transport model for shale gas reservoirs including non-darcy flow effects, in: SPE Annual Technical Conference and Exhibition, Society of Petroleum Engineers, 2012.

[24] H. Singh, F. Javadpour, A. Ettehadtavakkol, H. Darabi, Nonempirical apparent permeability of shale, SPE Reservoir Evaluation \& Engineering 17 (03) (2014) 414-424.

[25] K. Wu, Apparent permeability for gas flow in shale reservoirs coupling effects of gas diffusion and desorption, in: Unconventional Resources Technology Conference (URTEC), Society of Petroleum Engineers, 2014.

[26] J. Klaver, G. Desbois, J. L. Urai, R. Littke, Bib-sem study of the pore space morphology in early mature posidonia shale from the hils area, germany, International Journal of Coal Geology 103 (2012) 12-25.

[27] J. Klaver, G. Desbois, R. Littke, J. L. Urai, Bib-sem characterization of pore space morphology and distributions in postmature to overmature samples from the haynesville and bossier shales, Marine and Petroleum Geology 59 (2015) 451-466.

[28] R. M. Slatt, N. R. O'Brien, Pore types in the barnett and woodford gas shales: Contribution to understanding gas storage and migration pathways in fine-grained rocks, AAPG bulletin 95 (12) (2011) 20172030 .

[29] M. Milner, R. McLin, J. Petriello, Imaging texture and porosity in mudstones and shales: Comparison of secondary and ion-milled backscatter 
sem methods, in: Canadian Unconventional Resources and International Petroleum Conference, Society of Petroleum Engineers, 2010.

[30] M. E. Curtis, R. J. Ambrose, C. H. Sondergeld, Structural characterization of gas shales on the micro-and nano-scales, in: Canadian Unconventional Resources and International Petroleum Conference, Society of Petroleum Engineers, 2010.

[31] G. R. McNamara, G. Zanetti, Use of the boltzmann equation to simulate lattice-gas automata, Physical Review Letters 61 (20) (1988) 23322335 .

[32] F. Higuera, J. Jimenez, Boltzmann approach to lattice gas simulations, EPL (Europhysics Letters) 9 (7) (1989) 663-668.

[33] X. He, L. Luo, A priori derivation of the lattice boltzmann equation, Physical Review E 55 (6) (1997) R6333-R6336.

[34] X. He, L. Luo, Theory of the lattice boltzmann method: From the boltzmann equation to the lattice boltzmann equation, Physical Review E 56 (6) (1997) 6811-6817.

[35] A. Nabovati, E. W. Llewellin, A. Sousa, A general model for the permeability of fibrous porous media based on fluid flow simulations using the lattice boltzmann method, Composites Part A: Applied Science and Manufacturing 40 (6) (2009) 860-869.

[36] Z. Guo, T. Zhao, Lattice boltzmann model for incompressible flows through porous media, Physical Review E 66 (3) (2002) 036304.

[37] C. Pan, M. Hilpert, C. Miller, Lattice-boltzmann simulation of twophase flow in porous media, Water Resources Research 40 (1) (2004) w01501.

[38] A. K. Gunstensen, D. H. Rothman, Lattice-boltzmann studies of immiscible two-phase flow through porous media, Journal of Geophysical Research: Solid Earth (1978-2012) 98 (B4) (1993) 6431-6441.

[39] S. Succi, E. Foti, F. Higuera, Three-dimensional flows in complex geometries with the lattice boltzmann method, EPL (Europhysics Letters) 10 (5) (1989) 433-438. 
[40] Q. Kang, D. Zhang, S. Chen, X. He, Lattice boltzmann simulation of chemical dissolution in porous media, Physical Review E 65 (3) (2002) 036318 .

[41] Q. Kang, P. C. Lichtner, D. Zhang, Lattice boltzmann pore-scale model for multicomponent reactive transport in porous media, Journal of Geophysical Research: Solid Earth (1978-2012) 111 (B5) (2006) B05203.

[42] M. L. Porter, M. G. Schaap, D. Wildenschild, Lattice-boltzmann simulations of the capillary pressure-saturation-interfacial area relationship for porous media, Advances in Water Resources 32 (11) (2009) 16321640 .

[43] S. Ramaswamy, M. Gupta, A. Goel, U. Aaltosalmi, M. Kataja, A. Koponen, B. Ramarao, The 3d structure of fabric and its relationship to liquid and vapor transport, Colloids and Surfaces A: Physicochemical and Engineering Aspects 241 (1) (2004) 323-333.

[44] T. Koido, T. Furusawa, K. Moriyama, An approach to modeling twophase transport in the gas diffusion layer of a proton exchange membrane fuel cell, Journal of Power Sources 175 (1) (2008) 127-136.

[45] L. Wang, B. Afsharpoya, Modeling fluid flow in fuel cells using the lattice-boltzmann approach, Mathematics and Computers in Simulation 72 (2) (2006) 242-248.

[46] N. S. Martys, H. Chen, Simulation of multicomponent fluids in complex three-dimensional geometries by the lattice boltzmann method, Physical Review E 53 (1) (1996) 743-751.

[47] B. Ferreol, D. H. Rothman, Lattice-boltzmann simulations of flow through fontainebleau sandstone, in: Multiphase Flow in Porous Media, Springer, 1995, pp. 3-20.

[48] E. S. Boek, M. Venturoli, Lattice-boltzmann studies of fluid flow in porous media with realistic rock geometries, Computers \& Mathematics with Applications 59 (7) (2010) 2305-2314.

[49] M. Coles, R. Hazlett, E. Muegge, K. Jones, B. Andrews, B. Dowd, P. Siddons, A. Peskin, P. Spanne, W. Soll, Developments in synchrotron x-ray microtomography with applications to flow in porous 
media, in: SPE Annual Technical Conference and Exhibition, Society of Petroleum Engineers, 1996.

[50] B. Li, D. Kwok, Li and kwok reply:, Physical Review Letters 92 (2004) 139402.

[51] T. Lee, C. Lin, Rarefaction and compressibility effects of the latticeboltzmann-equation method in a gas microchannel, Physical Review E 71 (4) (2005) 046706.

[52] X. Niu, C. Shu, Y. Chew, A lattice boltzmann bgk model for simulation of micro flows, EPL (Europhysics Letters) 67 (4) (2004) 600-606.

[53] M. Sbragaglia, S. Succi, Analytical calculation of slip flow in lattice boltzmann models with kinetic boundary conditions, Physics of Fluids (1994-present) 17 (9) (2005) 093602.

[54] S. Succi, Mesoscopic modeling of slip motion at fluid-solid interfaces with heterogeneous catalysis, Physical Review Letters 89 (6) (2002) 064502 .

[55] G. Tang, W. Tao, Y. He, Lattice boltzmann method for simulating gas flow in microchannels, International Journal of Modern Physics $\mathrm{C}$ 15 (02) (2004) 335-347.

[56] Y. Zhang, R. Qin, D. R. Emerson, Lattice boltzmann simulation of rarefied gas flows in microchannels, Physical Review E 71 (4) (2005) 047702 .

[57] Z. Guo, T. Zhao, Y. Shi, Physical symmetry, spatial accuracy, and relaxation time of the lattice boltzmann equation for microgas flows, Journal of Applied physics 99 (7) (2006) 074903.

[58] F. Verhaeghe, L. Luo, B. Blanpain, Lattice boltzmann modeling of microchannel flow in slip flow regime, Journal of Computational Physics 228 (1) (2009) 147-157.

[59] A. Homayoon, A. Isfahani, E. Shirani, M. Ashrafizadeh, A novel modified lattice boltzmann method for simulation of gas flows in wide range of knudsen number, International Communications in Heat and Mass Transfer 38 (6) (2011) 827-832. 
[60] Z. Guo, C. Zheng, B. Shi, Lattice boltzmann equation with multiple effective relaxation times for gaseous microscale flow, Physical Review E 77 (3) (2008) 036707.

[61] C. Zhuo, C. Zhong, Filter-matrix lattice boltzmann model for microchannel gas flows, Physical Review E 88 (5) (2013) 053311.

[62] Q. Li, Y. He, G. Tang, W. Tao, Lattice boltzmann modeling of microchannel flows in the transition flow regime, Microfluidics and Nanofluidics 10 (3) (2011) 607-618.

[63] G. Tang, Y. Zhang, X. Gu, D. Emerson, Lattice boltzmann modelling knudsen layer effect in non-equilibrium flows, EPL (Europhysics Letters) 83 (4) (2008) 40008.

[64] H. Shokouhmand, A. Meghdadi Isfahani, An improved thermal lattice boltzmann model for rarefied gas flows in wide range of knudsen number, International Communications in Heat and Mass Transfer 38 (10) (2011) 1463-1469.

[65] X. Niu, C. Shu, Y. Chew, Numerical simulation of isothermal micro flows by lattice boltzmann method and theoretical analysis of the diffuse scattering boundary condition, International Journal of Modern Physics C 16 (12) (2005) 1927-1941.

[66] G. Tang, W. Tao, Y. He, Lattice boltzmann method for gaseous microflows using kinetic theory boundary conditions, Physics of Fluids 17 (5) (2005) 058101.

[67] L. Luo, Comment on"heat transfer and fluid flow in microchannels and nanochannels at high knudsen number using thermal lattice-boltzmann method", Physical Review E 84 (2011) 048301.

[68] A. Norouzi, J. A. Esfahani, Two relaxation time lattice boltzmann equation for high knudsen number flows using wall function approach, Microfluidics and Nanofluidics (2014) 1-10.

[69] J. A. Esfahani, A. Norouzi, Two relaxation time lattice boltzmann model for rarefied gas flows, Physica A: Statistical Mechanics and its Applications 393 (2014) 51-61. 
[70] X. Zhang, L. Xiao, X. Shan, L. Guo, Lattice boltzmann simulation of shale gas transport in organic nano-pores, Scientific reports 4.

[71] K. Suga, Lattice boltzmann methods for complex micro-flows: applicability and limitations for practical applications, Fluid Dynamics Research 45 (3) (2013) 034501.

[72] C. K. Aidun, J. R. Clausen, Lattice-boltzmann method for complex flows, Annual Review of Fluid Mechanics 42 (2010) 439-472.

[73] Z. Guo, C. Zheng, B. Shi, Discrete lattice effects on the forcing term in the lattice boltzmann method, Physical Review E 65 (4) (2002) 046308.

[74] S. H. Kim, H. Pitsch, I. D. Boyd, Accuracy of higher-order lattice boltzmann methods for microscale flows with finite knudsen numbers, Journal of Computational Physics 227 (19) (2008) 8655-8671.

[75] A. P. Randles, V. Kale, J. Hammond, W. Gropp, E. Kaxiras, Performance analysis of the lattice boltzmann model beyond navier-stokes, in: Parallel \& Distributed Processing (IPDPS), 2013 IEEE 27th International Syoposium, IEEE, 2013.

[76] P. L. Bhatnagar, E. P. Gross, M. Krook, A model for collision processes in gases. i. small amplitude processes in charged and neutral one-component systems, Physical Review 94 (3) (1954) 511-525.

[77] P. Lallemand, L.-S. Luo, Theory of the lattice boltzmann method: Dispersion, dissipation, isotropy, galilean invariance, and stability, Physical Review E 61 (6) (2000) 6546-6562.

[78] L. Luo, W. Liao, X. Chen, Y. Peng, W. Zhang, Numerics of the lattice boltzmann method: Effects of collision models on the lattice boltzmann simulations, Physical Review E 83 (5) (2011) 056710.

[79] I. Ginzburg, Equilibrium-type and link-type lattice boltzmann models for generic advection and anisotropic-dispersion equation, Advances in Water resources 28 (11) (2005) 1171-1195.

[80] D. C. Tretheway, C. D. Meinhart, Apparent fluid slip at hydrophobic microchannel walls, Physics of Fluids (1994-present) 14 (3) (2002) L9L12. 
[81] J. C. Harley, Y. Huang, H. H. Bau, J. N. Zemel, Gas flow in microchannels, Journal of Fluid Mechanics 284 (1995) 257-274.

[82] L. Zhu, D. Tretheway, L. Petzold, C. Meinhart, Simulation of fluid slip at $3 \mathrm{~d}$ hydrophobic microchannel walls by the lattice boltzmann method, Journal of Computational Physics 202 (1) (2005) 181-195.

[83] J. Harting, C. Kunert, H. J. Herrmann, Lattice boltzmann simulations of apparent slip in hydrophobic microchannels, EPL (Europhysics Letters) 75 (2) (2006) 328.

[84] R. Benzi, L. Biferale, M. Sbragaglia, S. Succi, F. Toschi, Mesoscopic two-phase model for describing apparent slip in micro-channel flows, EPL (Europhysics Letters) 74 (4) (2006) 651.

[85] Y. Zhang, X. Gu, R. W. Barber, D. R. Emerson, Capturing knudsen layer phenomena using a lattice boltzmann model, Physical Review E 74 (4) (2006) 046704.

[86] S. Succi, The Lattice-Boltzmann Equation, Oxford university press, Oxford, 2001.

[87] X. Nie, G. D. Doolen, S. Chen, Lattice-boltzmann simulations of fluid flows in mems, Journal of Statistical Physics 107 (1-2) (2002) 279-289.

[88] C. Lim, C. Shu, X. Niu, Y. Chew, Application of lattice boltzmann method to simulate microchannel flows, Physics of Fluids (1994present) 14 (7) (2002) 2299-2308.

[89] Z. Chai, Z. Guo, L. Zheng, B. Shi, Lattice boltzmann simulation of surface roughness effect on gaseous flow in a microchannel, Journal of Applied Physics 104 (1) (2008) 014902.

[90] S. Tao, Z. Guo, Boundary condition for lattice boltzmann modeling of microscale gas flows with curved walls in the slip regime, Physical Review E 91 (4) (2015) 043305.

[91] D. Technischen, Lattice boltzmann simulations in the finite knudsen number range within the peano framework, Ph.D. thesis, University At Munchen (2011). 
[92] S. Ansumali, I. V. Karlin, Kinetic boundary conditions in the lattice boltzmann method, Physical Review E 66 (2) (2002) 026311.

[93] X. Niu, S. A. Hyodo, T. Munekata, K. Suga, Kinetic lattice boltzmann method for microscale gas flows: Issues on boundary condition, relaxation time, and regularization, Physical Review E 76 (3) (2007) 036711.

[94] Z. Chai, B. Shi, Z. Guo, J. Lu, Gas flow through square arrays of circular cylinders with klinkenberg effect: a lattice boltzmann study, Communications in Computational Physics 8 (5) (2010) 1052.

[95] Z. Guo, C. Zheng, Analysis of lattice boltzmann equation for microscale gas flows: relaxation times, boundary conditions and the knudsen layer, International Journal of Computational Fluid Dynamics 22 (7) (2008) 465-473.

[96] Z. Guo, B. Shi, T.-S. Zhao, C. Zheng, Discrete effects on boundary conditions for the lattice boltzmann equation in simulating microscale gas flows, Physical Review E 76 (5) (2007) 056704.

[97] P. Neumann, T. Rohrmann, Lattice boltzmann simulations in the slip and transition flow regime with the peano framework, Open Journal of Fluid Dynamics 2 (2012) 101-110.

[98] X. He, Q. Zou, L. Luo, M. Dembo, Analytic solutions of simple flows and analysis of nonslip boundary conditions for the lattice boltzmann bgk model, Journal of Statistical Physics 87 (1-2).

[99] L. Zheng, Z. Guo, B. Shi, Microscale boundary conditions of the lattice boltzmann equation method for simulating microtube flows, Physical Review E 86 (1) (2012) 016712.

[100] J. C. Maxwell, On stresses in rarified gases arising from inequalities of temperature, Philosophical Transactions of the royal society of London 170 (1879) 231-256.

[101] W. Zhang, G. Meng, X. Wei, A review on slip models for gas microflows, Microfluidics and Nanofluidics 13 (6) (2012) 845-882. 
[102] B. C. Eu, R. E. Khayat, G. D. Billing, C. Nyeland, Nonlinear transport coefficients and plane couette flow of a viscous, heat-conducting gas between two plates at different temperatures, Canadian Journal of Physics 65 (9) (1987) 1090-1103.

[103] R. Myong, A generalized hydrodynamic computational model for rarefied and microscale diatomic gas flows, Journal of Computational Physics 195 (2) (2004) 655-676.

[104] Q. Yang, H. Zhang, Discussion on the gaseous slip model based on langmuir adsorption isotherm, Physics Procedia 32 (2012) 179-183.

[105] H. M. Kim, D. Kim, W. T. Kim, P. Seung Chung, M. S. Jhon, Langmuir slip model for air bearing simulation using the lattice boltzmann method, Magnetics, IEEE Transactions on 43 (6) (2007) 2244-2246.

[106] S. Chen, Z. Tian, Simulation of microchannel flow using the lattice boltzmann method, Physica A: Statistical Mechanics and its Applications 388 (23) (2009) 4803-4810.

[107] X. Shan, X. Yuan, H. Chen, Kinetic theory representation of hydrodynamics: a way beyond the navier-stokes equation, Journal of Fluid Mechanics 550 (2006) 413-441.

[108] N. Dongari, Y. Zhang, J. M. Reese, Modeling of knudsen layer effects in micro/nanoscale gas flows, Journal of Fluids Engineering 133 (7) (2011) 071101.

[109] C. Cercignani, A. Daneri, Flow of a rarefied gas between two parallel plates, Journal of Applied Physics 34 (12) (1963) 3509-3513.

[110] Y. Zhang, R. Qin, Y. Sun, R. Barber, D. Emerson, Gas flow in microchannels-a lattice boltzmann method approach, Journal of Statistical Physics 121 (1-2) (2005) 257-267.

[111] L. O'Hare, D. A. Lockerby, J. M. Reese, D. R. Emerson, Near-wall effects in rarefied gas micro-flows: some modern hydrodynamic approaches, International journal of hear and fluid flow 28 (1) (2007) $37-43$. 
[112] D. Stops, The mean free path of gas molecules in the transition regime, Journal of Physics D: Applied Physics 3 (5) (1970) 685-696.

[113] E. J. Arlemark, S. K. Dadzie, J. M. Reese, An extension to the navierstokes equations to incorporate gas molecular collisions with boundaries, Journal of Heat Transfer 132 (4) (2010) 041006.

[114] V. K. Michalis, A. N. Kalarakis, E. D. Skouras, V. N. Burganos, Rarefaction effects on gas viscosity in the knudsen transition regime, Microfluidics and Nanofluidics 9 (4-5) (2010) 847-853.

[115] Y. Sun, W. Chan, Analytical modeling of rarefied poiseuille flow in microchannels, Journal of Vacuum Science \& Technology A 22 (2) (2004) 383-394.

[116] A. Kalarakis, V. Michalis, E. Skouras, V. Burganos, Mesoscopic simulation of rarefied flow in narrow channels and porous media, Transport in Porous Media 94 (1) (2012) 385-398.

[117] S. Ansumali, I. Karlin, S. Arcidiacono, A. Abbas, N. Prasianakis, Hydrodynamics beyond navier-stokes: Exact solution to the lattice boltzmann hierarchy, Physical Review Letters 98 (12) (2007) 124502.

[118] R. Zhang, X. Shan, H. Chen, Efficient kinetic method for fluid simulation beyond the navier-stokes equation, Physical Review E 74 (4) (2006) 046703.

[119] S. S. Chikatamarla, I. V. Karlin, Entropy and galilean invariance of lattice boltzmann theories, Physical Review Letters 97 (19) (2006) 190601.

[120] L. Szalmás, Knudsen layer theory for high-order lattice boltzmann models, Europhysics Letters 80 (2) (2007) 24003.

[121] J. Meng, Y. Zhang, Gauss-hermite quadratures and accuracy of lattice boltzmann models for nonequilibrium gas flows, Physical Review E 83 (3) (2011) 036704.

[122] J. Meng, Y. Zhang, Accuracy analysis of high-order lattice boltzmann models for rarefied gas flows, Journal of Computational Physics 230 (3) (2011) 835-849. 
[123] K. Suga, S. Takenaka, T. Ito, M. Kaneda, T. Kinjo, S. Hyodo, Evaluation of a lattice boltzmann method in a complex nanoflow, Physical Review E 82 (1) (2010) 016701.

[124] K. Suga, S. Takenaka, T. Ito, M. Kaneda, T. Kinjo, S. Hyodo, Lattice boltzmann flow simulation in micro-nano transitional porous media, in: 2010 14th International Heat Transfer Conference, American Society of Mechanical Engineers, 2010, pp. 321-329.

[125] Y. Wu, J. Li, D. Ding, C. Wang, Y. Di, A generalized framework model for the simulation of gas production in unconventional gas reservoirs, SPE Journal 19 (05) (2014) 845-857.

[126] Y. Li, Y. Jiang, J. Zhao, C. Liu, L. Zhang, Extended finite element method for analysis of multi-scale flow in fractured shale gas reservoirs, Environmental Earth Sciences 73 (10) (2015) 6035-6045.

[127] M. Sheng, G. Li, S. N. Shah, X. Jin, Extended finite element modeling of multi-scale flow in fractured shale gas reservoirs, in: SPE Annual Technical Conference and Exhibition, Society of Petroleum Engineers, 2012 .

[128] N. Gholizadeh Doonechaly, R. Abdel Azim, S. S. Rahman, Evaluation of recoverable energy potential from enhanced geothermal systems: A sensitivity analysis in a poro-thermo-elastic framework, Geofluids.

[129] Z. Pan, L. D. Connell, Reservoir simulation of free and adsorbed gas production from shale, Journal of Natural Gas Science and Engineering 22 (2015) 359-370.

[130] C. J. Landry, Matrix-fracture connectivity in eagle ford shale, in: Unconventional Resources Technology Conference (URTEC), Society of Petroleum Engineers, 2014.

[131] P. Tiwari, M. Deo, C. Lin, J. Miller, Characterization of oil shale pore structure before and after pyrolysis by using x-ray micro ct, Fuel 107 (2013) 547-554.

[132] J. Ma, A multi-scale framework for digital core analysis of gas shale at millimeter scales, in: Unconventional Resources Technology Conference (URTEC), Society of Petroleum Engineers, 2014. 
[133] C. Chen, D. Hu, D. Westacott, D. Loveless, Nanometer-scale characterization of microscopic pores in shale kerogen by image analysis and pore-scale modeling, Geochemistry, Geophysics, Geosystems 14 (10) (2013) 4066-4075.

[134] C. Chen, D. Hu, V. N. Martysevich, Applications of high-resolution imaging and high-performance parallel computing in unconventional energy recovery, in: Abu Dhabi International Petroleum Exhibition and Conference, Society of Petroleum Engineers, 2014.

[135] M. T. Cantisano, Relative permeability in a shale formation in colombia using digital rock physics, in: Unconventional Resources Technology Conference (URTEC), Society of Petroleum Engineers, 2013.

[136] N. Nagarajan, Critical role of rock and fluid-impact on reservoir performance on unconventional shale reservoirs, in: Unconventional Resources Technology Conference (URTEC), Society of Petroleum Engineers, 2013.

[137] L. Klinkenberg, The permeability of porous media to liquids and gases, in: Drilling and Production Practice, American Petroleum Institute, 1941.

[138] A. M. Allan, G. Mavko, The effect of adsorption and knudsen diffusion on the steady-state permeability of microporous rocks, Geophysics 78 (2) (2013) D75-D83.

[139] L. Chen, L. Zhang, Q. Kang, J. Yao, W. Tao, Nanoscale simulation of shale transport properties using the lattice boltzmann method: permeability and diffusivity, Scientific Reports.

[140] A. S. Ziarani, R. Aguilera, Knudsens permeability correction for tight porous media, Transport in Porous Media 91 (1) (2012) 239-260.

[141] L. Chen, Q. Kang, R. Pawar, Y.-L. He, W. Tao, Pore-scale prediction of transport properties in reconstructed nanostructures of organic matter in shales, Fuel 158 (0) (2015) 650-658.

[142] M. Kazemi, A. Takbiri-Borujeni, An analytical model for shale gas permeability, International Journal of Coal Geology 146 (2015) 188197. 
[143] E. Fathi, A. Tinni, I. Y. Akkutlu, Correction to klinkenberg slip theory for gas flow in nano-capillaries, International Journal of Coal Geology 103 (2012) 51-59.

[144] X. Shan, H. Chen, Lattice boltzmann model for simulating flows with multiple phases and components, Physical Review E 47 (3) (1993) $1815-1820$.

[145] Z. Li, T. Min, L. Chen, Q. Kangd, Y.-L. He, W.-Q. Tao, Investigation of methane adsorption and its effect on gas transport in shale matrix through microscale and mesoscale simulations, arXiv preprint arXiv:1503.07445.

[146] J. Ren, P. Guo, Z. Guo, Z. Wang, A lattice boltzmann model for simulating gas flow in kerogen pores, Transport in Porous Media (2014) $1-17$.

[147] O. Dardis, J. McCloskey, Lattice boltzmann scheme with real numbered solid density for the simulation of flow in porous media, Physical Review E 57 (4) (1998) 4834.

[148] M. A. Spaid, F. R. Phelan Jr, Lattice boltzmann methods for modeling microscale flow in fibrous porous media, Physics of Fluids (1994present) 9 (9) (1997) 2468-2474.

[149] Q. Kang, D. Zhang, S. Chen, Unified lattice boltzmann method for flow in multiscale porous media, Physical Review E 66 (5) (2002) 056307.

[150] L. Chen, W. Fang, Q. Kang, J. D. Hyman, H. S. Viswanathan, W.Q. Tao, Generalized lattice boltzmann model for flow through tight porous media with klinkenberg's effect, Physical Review E 91 (3) (2015) 033004 .

[151] P. Carman, Fluid flow through granular beds, Chemical Engineering Research and Design 75 (1997) S32-S48.

[152] J. Heid, J. McMahon, R. Nielsen, S. Yuster, Study of the permeability of rocks to homogeneous fluids, in: Drilling and production practice, American Petroleum Institute, 1950. 
1179 [153] L. Chen, Q. Kang, Z. Dai, H. S. Viswanathan, W. Tao, Permeability prediction of shale matrix reconstructed using the elementary building block model, Fuel 160 (2015) 346-356. 